\title{
Influence of the Soret effect on convection of binary fluids
}

\author{
St. Hollinger and M. Lücke \\ Institut für Theoretische Physik, Universität des Saarlandes, Postfach 151150, \\ D-66041 Saarbrücken, Germany
}

\begin{abstract}
Convection in horizontal layers of binary fluids heated from below and in particular the influence of the Soret effect on the bifurcation properties of extended stationary and traveling patterns that occur for negative Soret coupling is investigated theoretically. The fixed points corresponding to these two convection structures are determined for realistic boundary conditions with a many mode Galerkin scheme for temperature and concentration and an accurate one mode truncation of the velocity field. This solution procedure yields the stable and unstable solutions for all stationary and traveling patterns so that complete phase diagrams for the different convection types in typical binary liquid mixtures can easily be computed. Also the transition from weakly to strongly nonlinear states can be analyzed in detail. An investigation of the concentration current and of the relevance of its constituents shows the way for a simplification of the mode representation of temperature and concentration field as well as for an analytically manageable few mode description.
\end{abstract}

PACS number(s): 47.20.-k, 47.10.+g, 51.10.+y 


\section{INTRODUCTION}

Convection in binary miscible fluids like, e. g., ethanol--water or ${ }^{3} \mathrm{He}^{-}{ }^{4} \mathrm{He}$ is a well established and accepted system for studying instabilities, bifurcations, complex spatiotemporal behaviour, and turbulence. This is on the one hand due to its sufficiently simple experimental realization under well controllable conditions. On the other hand, a great advantage for the theoretical analysis is the solid knowledge of the governing field equations. So, recently a lot of research activities [1] [16] have been directed towards investigating the enormous variety of pattern forming behaviour in this system. The richness of spatio temporal phenomena in binary fluid mixtures stems from a feed-back loop between the fields of velocity, concentration, and temperature. Let us start with the velocity field: The convective flow is driven by the buoyancy force field which itself is determined by variations of the temperature and of the concentration field. The latter are on the one hand generated via the thermodiffusive Soret effect by temperature gradients and on the other hand they are reduced by concentration diffusion and by mixing due to the convective flow. Since these changes influence the buoyancy which drives the flow the feed back loop is closed.

In this article we concentrate on the Soret coupling and its influence on spatially extended convection states of straight parallel rolls that occur either as a horizontally traveling wave (TW) or in the form of stationary "overturning" convection (SOC) rolls. Among others, we elucidate the Soret induced changes in the combined SOC-TW bifurcation topology which offers in both types of convection the possibility of sub- and supercritically bifurcating branches depending on the strength of the Soret coupling. Both solution branches develop with increasing Soret coupling saddle node bifurcations which give rise to stability changes. Finally, there exists a merging point of the SOC and the TW branches for moderate negative Soret couplings. This competition of stationary and traveling states can only be observed for negative Soret coupling, where temperature gradients induce adverse concentration gradients that stabilize the unstable thermal layering. For positive Soret couplings, there is no oscillatory instability of the basic state. The two interesting cases for negative coupling are 
now weak and strong Soret effect. For the latter one a bistability of slow and fast TWs was recently reported [17] which coexist with the likewise stable basic state of heat conduction. A detailed phase diagram was discussed [17] describing the Soret dependence of the saddle nodes. For weak Soret couplings, however, a detailed study of the bifurcation toplogy was missing. Data from direct numerical simulations are sparse since in the vicinity of saddle nodes and bifurcation points the intrinsic time scale of the system is arbitrarily long. Thus, phase diagrams to elucidate the whole bifurcation topology were incomplete.

We have determined the SOC and TW fixed points of the system by a many mode Galerkin scheme whose convergence properties do not depend on the time scale of the system. In particular, this allowed us to study all unstable branches on which, especially, the transition from weakly to strongly nonlinear convection takes place. Furthermore, a detailed explanation of the concentration distribution and its relation to convection is given.

Our article is organized as follows: The second section presents the system, the fields needed for its description, their governing equations with the explanation of the relevant fluid and control parameters. Finally, it presents a short survey on the typical bifurcation scenario in the convection of binary liquid mixtures with negative Soret coupling. The third section shows the field truncations, the method of solving the system and the solutions of our many mode Galerkin scheme basing on a reasonable approximation in the velocity field. In the fourth section we discuss the influence of the Soret coupling on the bifurcation topology by means of exemplary bifurcation diagrams realized in experimentally feasible mixtures and a detailed phase diagram. Furthermore, evidence for an instability of a TW towards a modulated TW (MTW) is given. The fluid parameter range for its occurence is elucidated for ethanol-water as well as for ${ }^{3} \mathrm{He}-{ }^{4} \mathrm{He}-$ mixtures. Finally, we extract the relation between concentration distribution and convective structure and we investigate the importance of the Soret effect at the boundaries and its neglibility in the bulk. 


\section{SYSTEM}

A layer of a binary fluid mixture with a mean temperature $\bar{T}$ and a mean concentration $\bar{C}$ is confined between two perfectly heat conducting and impervious plates separated by a distance $d$ and exposed to a vertical, homogeneous gravitational acceleration $g$. The lower (upper) plate is kept at a fixed temperature $\bar{T}+\Delta T / 2(\bar{T}-\Delta T / 2)$.

The fluid parameters are $\rho$ (density of the fluid), $\alpha=-\frac{1}{\rho} \frac{\partial \rho}{\partial T}$ (thermal expansion co-

efficient), $\beta=-\frac{1}{\rho} \frac{\partial \rho}{\partial \bar{C}}$ (solutal expansion coefficient), $\nu$ (kinematic viscosity), $\kappa$ (thermal diffusivity), $k_{T}$ (thermodiffusion coefficient), and $D$ (solutal diffusivity).

\section{A. Scaling and balance equations}

We scale lengths by the height $d$ of the layer, times by the vertical diffusion time $d^{2} / \kappa$ of the heat and accordingly velocities by $\kappa / d$. The deviation $T$ of the temperature from its mean $\bar{T}$ is reduced by $\Delta T$, that of the concentration field by $\frac{\alpha}{\beta} \Delta T$, and the pressure $p$ by $\frac{\rho \kappa^{2}}{d^{2}}$. Then, the balance equations for mass, momentum, heat, and concentration [2, [3] read in Oberbeck-Boussinesq approximation 18

$$
\begin{aligned}
0= & -\boldsymbol{\nabla} \cdot \mathbf{u} \\
\partial_{t} \mathbf{u}= & -(\mathbf{u} \cdot \boldsymbol{\nabla}) \mathbf{u}-\boldsymbol{\nabla}\left[p+\left(\frac{d^{3}}{\kappa^{2}} g\right) z\right] \\
& +\sigma \nabla^{2} \mathbf{u}+R \sigma(T+C) \mathbf{e}_{z} \\
\partial_{t} T= & -\boldsymbol{\nabla} \cdot \mathbf{Q}=-(\mathbf{u} \cdot \boldsymbol{\nabla}) T+\nabla^{2} T \\
\partial_{t} C= & -\boldsymbol{\nabla} \cdot \mathbf{J}=-(\mathbf{u} \cdot \boldsymbol{\nabla}) C+L \nabla^{2}(C-\psi T) .
\end{aligned}
$$

The Dufour effect describing currents of heat driven by concentration gradients is discarded in (2.1d) since it is relevant only in binary gas mixtures [18,19] or in liquids near the critical point 20.

The dimensionless fluid parameters are the Prandtl number $\sigma=\nu / \kappa$, the Lewis number $L=D / \kappa$, and the separation ratio $\psi=-\frac{\beta}{\alpha} \frac{k_{T}}{T}$. The latter characterizes the strength of the 
Soret effect. The Rayleigh number $R=\frac{\alpha g d^{3}}{\nu \kappa} \Delta T$ serves as control parameter measuring the thermal stress.

\section{B. Bounday conditions}

We use experimentally realized boundary conditions for the top and bottom plates at $z= \pm 1 / 2$ which are no slip for the velocity field,

$$
\mathbf{u}(x, y, z= \pm 1 / 2 ; t)=0
$$

perfectly heat conducting for the temperature field,

$$
T(x, y, z= \pm 1 / 2 ; t)=\mp 1 / 2
$$

and impermeable for the concentration field, i. e.

$$
\mathbf{e}_{z} \cdot \mathbf{J}=-L \partial_{z}(C-\psi T)(x, y, z= \pm 1 / 2 ; t)=0
$$

We restrict ourselves to the description of extended roll like patterns that are homogeneous in one lateral direction say $y$. So, we investigate two dimensional states of a certain lateral periodicity length $\lambda=2 \pi / k$. In most cases we take $k=\pi$, i. e., $\lambda$ twice the thickness of the fluid layer, which is close to the critical wavelengths for the negative Soret couplings investigated here. Furthermore, the stable nonlinear TW and SOC states that are observed in experiments have typically a wave number $k=\pi$.

\section{Conductive state}

In the motionless basic state, a vertically linear temperature profile, $T_{\text {cond }}=-z$, is observed due to the different top and bottom temperature. This leads via the Soret effect and the no flux condition for $\mathbf{J}$ to a likewise linear concentration profile, $C_{\text {cond }}=-\psi z$. Both together yield the hydrostatic pressure

$$
p_{\text {cond }}=p_{0}-\frac{1}{2} R \sigma(1+\psi) z^{2}-\left(\frac{d^{3}}{\kappa^{2}} g\right) z
$$

in the quiescent state. 


\section{Control and order parameters}

The dimensionless temperature difference between the two plates, namely the Rayleigh number $R$, is used as contol parameter. Mostly we scale it by the value of the onset of convection in a pure fluid:

$$
r=\frac{R}{R_{\mathrm{c}}^{0}}=\frac{R}{1707.762} .
$$

The convective states of the system are characterized by four order parameters:

(i) The maximum $w_{\max }$ of the vertical velocity field.

(ii) The Nusselt number $N=\left\langle\mathbf{Q} \cdot \mathbf{e}_{z}\right\rangle_{x}$ giving the lateral average of the vertical heat current through the system. In the basic state of heat conduction its value is 1 and larger than 1 in all convective states.

(iii) The variance $M=\sqrt{\left\langle C^{2}\right\rangle_{x, z} /\left\langle C_{\text {cond }}^{2}\right\rangle_{x, z}}$ of the concentration field being a measure for the mixing in the system. The better the fluid is mixed the more the concentration is globally equilibrated to its mean value 0 so that $M$ vanishes in optimally mixing, strongly convecting states.

(iv) The frequency $\omega$ of a traveling wave. Thus, extended TWs with a wave number $k$ have a phase velocity $v=\omega / k$. They are stationary states in a reference frame comoving with $v$ relative to the laboratory system.

\section{E. Typical bifurcation scenario}

For fluid parameters typically realized in mixtures of water and about 10 wt.\% ethanol, an oscillatory, subcritical onset of convection is observed. It is connected by an unstable TW branch with a saddle node bifurcation giving rise to stable, strongly nonlinear TW states. At a certain Rayleigh number, the phase velocity of these waves vanishes and the SOC branch of stable stationary states can be observed. Along the TW bifurcation branch which is shown in detail in Fig. 1 the concentration changes its structure from lateral homogeneity and vertically linear layering in the basic state over plateau-like distributions in 
fast TWs to boundary layer dominated, slowly traveling waves (see the discussion and the figures in Sec. (IVB). The contrast between two adjacent TW rolls is strongly related to the phase velocity of the TW and it vanishes with this velocity. So, SOCs do not show such a concentration contrast. In SOCs adjacent rolls are mirror images of each other and they are separated from another and from the top and bottom plate only by thin boundary layers. The latter are a typical phenomenon for convection of weakly diffusing scalars.

\section{COMPUTATION OF EXTENDED STATES}

\section{A. Modelling the velocity field}

In liquid binary mixtures like ethanol-water momentum diffuses approximately ten times faster than heat. This means that the Prandtl number $\sigma$ is of $O(10)$ so that the velocity field may be adiabatically eliminated. Then, the momentum balance (2.1b), say in vertical direction, reduces to the balance of the diffusive term $\sigma \nabla^{2} w$ and the buoyant term $R \sigma(T+C)$ with the latter containing no derivatives. Thus, in a stationary flow, either in the laboratory frame or in a comoving one, the amplitudes of higher lateral Fourier modes $\hat{w}_{n}$ of the vertical velocity field $w=\mathbf{u} \cdot \mathbf{e}_{z}$ scale at least like $\frac{1}{(n k)^{2}}$ so that they decrease rapidly and even faster than those of the temperature field. That is the reason why higher modes than the critical first lateral Fourier mode are not necessary for a good description of the velocity field. One can expect this to hold for all $\sigma \gtrsim 1$.

The next question deals with the role of the lateral mean of the velocity field, i. e., of its zeroth lateral Fourier mode. Continuity implies that only the lateral velocity field $u=\mathbf{u} \cdot \mathbf{e}_{x}$ can contain such a mean flow. In order to determine the relevance of a $z-$ dependent mean flow we compare its maximum with the two other velocities in the system: The maximal vertical flow velocity $w_{\max }$ and the phase velocity $v$ of TWs. Just at the onset of convection, the mean flow may be estimated [21] to scale with $w_{\max }^{2}$ and to be very small in comparison with $v$. Furthermore, mean flow and phase velocity have the same sign. In 
strongly nonlinear states, the ratio of flow velocity and mean flow is nearly $10^{3}$ [13], but has changed sign. Thus, the mean flow has a non-monotonous dependence on the phase and flow velocity whereas all other properties of TWs like mixing and heat transport vary monotonically. Hence, the mean flow cannot contribute systematically to these properties characterizing TWs sufficiently. This is the reason, together with the smallness of the mean flow, for ignoring it altogether.

The $z$-dependence of the critical velocity field is described in an adequate manner (see, e. g., [22,23]) by the first even Chandrasekhar function [24] $\mathcal{C}_{1}(z)$. Then, the velocity field of straight rolls with axes oriented in $y$-direction that are propagating with phase velocity $v$ in $x$-direction is described by

$$
\mathbf{u}(x, z ; t)=\frac{w_{\max }}{\mathcal{C}_{1}(0)}\left(\begin{array}{c}
-\frac{1}{k} \sin k(x-v t) \mathcal{C}_{1}^{\prime}(z) \\
0 \\
\cos k(x-v t) \mathcal{C}_{1}(z)
\end{array}\right)
$$

Herein, the phase is chosen so that the maximal vertical flow occurs at $t=0$ and $x=0$.

Fig. 2 checks in the first row the applicability of the ansatz (3.1) by plotting the contribution of modes in the vertical velocity $w$ which are not represented by (3.1) for two separation ratios $\psi$. The convective amplitude $w_{\max }$ was chosen as abscissa to quantify the nonlinearity of the states. As a measure for the strength of higher contributions to the vertical velocity the error

$$
\left(\frac{\left\langle w_{\mathrm{MAC}}^{2}\right\rangle_{x, z}-\left\langle w w_{\mathrm{MAC}}\right\rangle_{x, z}}{\left\langle w_{\mathrm{MAC}}^{2}\right\rangle_{x, z}+\left\langle w w_{\mathrm{MAC}}\right\rangle_{x, z}}\right)^{1 / 2}
$$

was computed where $w_{\text {MAC }}(x, z)$ denotes the velocity field calculated from the full field equations by means of a finite difference MAC scheme [16]. $w(x, z)$ is the one mode approximation (3.1) with the two velocities $v$ and $w_{\max }$ chosen such that the numerically obtained velocity $w_{\text {MAC }}(x, z)$ is fitted best or, equivalently, the error (3.2) is minimized. For both 
Soret coupling strengths shown in Fig. 2 the error of the ansatz (3.1) is smaller than $4 \%$ but increases as expected with the convective amplitude.

The bottom row of Fig. 2 shows the maximum of the mean flow $\langle u(x, z)\rangle_{x}$. For both separation ratios the mean flow is smaller than the convective amplitude by at least three orders of magnitude.

All in all, Fig. 2 2 justifies the approximations implied by the ansatz (3.1) for the velocity field. An important consequence of the fixed spatial structure of the velocity field is that all nonlinear terms in the balance equations contain now the same amplitude, namely $w_{\max }$, since all nonlinearities are convective ones. This is an enormous simplification for the theoretical analysis as we will see below.

\section{B. Galerkin expansion for temperature and concentration}

The temperature field $T(x, z ; t)$ is appropriately described by

$$
\begin{aligned}
T(x, z ; t)=-z & +\sum_{n=0}^{\infty} \sum_{m=1}^{\infty}\left[T_{2 n}^{2 m}(t) e^{-2 i n k x}+\text { c.c. }\right] \sqrt{2} \sin 2 m \pi z \\
& +\sum_{n=0}^{\infty} \sum_{m=0}^{\infty}\left[T_{2 n+1}^{2 m+1}(t) e^{-i(2 n+1) k x}+\text { c.c. }\right] \sqrt{2} \cos (2 m+1) \pi z .
\end{aligned}
$$

This representation incorporates the mirror glide symmetry

$$
\left\{\begin{array}{l}
T \\
C
\end{array}\right\}(x, z ; t)=-\left\{\begin{array}{l}
T \\
C
\end{array}\right\}\left(x+\frac{\lambda}{2},-z ; t\right)
$$

of TW and SOC states [13, 16].

The representation of the concentration field is more subtle because of its boundary condition (2.2) coupled to the temperature field. The straight forward solution is the introduction of the combined field

$$
\zeta(x, z ; t)=\frac{1}{\psi} C(x, z ; t)-T(x, z ; t)
$$


obeying the equation

$$
\partial_{t} \zeta=-(\mathbf{u} \cdot \nabla) \zeta+L \nabla^{2} \zeta-\nabla^{2} T
$$

and the boundary condition

$$
\partial_{z} \zeta(x, z= \pm 1 / 2 ; t)=0
$$

Note that the $\zeta$ field (3.5) differs by a scaling factor $1 / \psi$ from the field that has mostly been used so far, see, e. g., 25,21,19. An adequate trigonometric expansion is

$$
\begin{aligned}
\zeta(x, z, t) & =\sum_{n=0}^{\infty} \sum_{m=0}^{\infty}\left[\zeta_{2 n}^{2 m+1}(t) e^{-2 i n k x}+\text { c.c. }\right] \sqrt{2} \sin (2 m+1) \pi z \\
& +\sum_{n=0}^{\infty} \sum_{m=0}^{\infty}\left[\zeta_{2 n+1}^{2 m}(t) e^{-i(2 n+1) k x}+\text { c.c. }\right] \sqrt{2} \cos 2 m \pi z
\end{aligned}
$$

which also takes the mirror glide symmetry (3.4) into account.

The introduction of the combined field $\zeta$ was also motivated by the wish to fulfill the boundary condition (2.2) or (3.7) for the concentration field exactly. However, the formulation of the concentration balance in terms of the $\zeta$ field causes a severe theoretical drawback for small Lewis numbers $L$ and separation ratios $\psi$ of order $O(1)$, that are typically realized in liquid mixtures: $\zeta$ and $T$ have the same order of magnitude according to (3.5). But in the balance equation (3.6) for $\zeta$ the diffsuive term enters with weight $L=O(0.01)$ and the temperature with $O(1)$. This means that for an appropriate solution of (3.6) for a particular $\zeta$-mode temperature modes are necessary which are $1 / L$ times, i. e. about 100 times, smaller than the $\zeta$-mode under consideration. Despite the fact that a relevant contribution of higher temperature modes was not observed neither in experiments nor in simulations they are necessary in (3.6) if the $\zeta$-field is introduced and small Lewis numbers are considered. That is mainly the reason why earlier Galerkin approximations using the $\zeta$ field and only few temperature modes did not succeed in describing nonlinear TW convection in binary liquid mixtures [21,23,25, 27]. In binary gas mixtures with typically $L=O(1)$ this problem does not occur [19]. 


\section{Solution of the system of mode equations}

After projecting the balance equations for $T$ and $\zeta$ onto the bases used in the mode expansions (3.3) for $T$ and (3.8) for $\zeta$ one gets ordinary differential equations for the mode amplitudes of the temperature and the $\zeta$ field

$$
\mathbf{X}(t)=\left[T_{2 n}^{2(m+1)}(t), T_{2 n+1}^{2 m+1}(t), \zeta_{2 n}^{2 m+1}(t), \zeta_{2 n+1}^{2 m}(t)\right]^{\mathrm{T}}
$$

The column vector $\mathbf{X}$ is written here as the transpose of a row vector.

In the case of SOC the amplitudes are constant and have to be chosen real in order to be compatible with the velocity ansatz (3.1) for $v \equiv 0$. The flow amplitude $w_{\max }$ appears via (3.1) in the $T$ and $\zeta$ field equations via the convective nonlinearity and in addition as an inhomogeneous contribution $w \partial_{z} T_{\text {cond }}=-w$ from the conductive part, $T_{\text {cond }}=-z$, of the temperature field (3.3). Thus, the mode equations for SOC states take the form

$$
\underline{\underline{\mathcal{M}}}_{\mathrm{SOC}}\left(w_{\max }\right) \cdot \mathbf{X}=\mathbf{B}_{\mathrm{SOC}}\left(w_{\max }\right)
$$

Here, the matrix $\underline{\underline{\mathcal{M}}}_{\mathrm{SOC}}\left(w_{\max }\right)$ of mode coupling coefficients contains $w_{\max }$ from the convective nonlinearity. The momentum balance in (2.1b) provides the relation between the Rayleigh number $R$ and the velocity amplitude $w_{\max }$

$$
R=\frac{w_{\max }}{\mathbf{a} \cdot \mathbf{X}_{\mathrm{SOC}}\left(w_{\max }\right)}
$$

containing the solution $\mathbf{X}_{\mathrm{SOC}}=\underline{\underline{\mathcal{M}}}_{\mathrm{SOC}}^{-1} \cdot \mathbf{B}_{\mathrm{SOC}}$ of (3.9) depending on $w_{\max }$. The vector a contains all projection coefficients. The pressure gradient in the momentum balance of (2.1b) may be eliminated by taking the curl of the balance equation. The nonlinearity of (2.1b) vanishes in the projection procedure when using only one velocity mode. Now, the solution procedure is obvious: Solving the linear system (3.9) for a given convective amplitude and inserting the result into (3.10) yields the Rayleigh number of that SOC state with the convective amplitude $w_{\max }$. Thus, the bifurcation diagram $R\left(w_{\max }\right)$ or $w_{\max }(R)$ may be calculated. 
Finding the TW solution is slightly more difficult since the modes are time dependent:

$$
\mathbf{X}_{\mathrm{TW}}(t)=\left[\widehat{T}_{2 n}^{2(m+1)} e^{i 2 n \omega t}, \widehat{T}_{2 n+1}^{2 m+1} e^{i(2 n+1) \omega t}, \widehat{\zeta}_{2 n}^{2 m+1} e^{i 2 n \omega t}, \widehat{\zeta}_{2 n+1}^{2 m} e^{i(2 n+1) \omega t}\right]^{\mathrm{T}}
$$

The form of the time dependence is determined by the fact that the TWs are stationary in a frame comoving with $v=\omega / k$. Therefore, the angular frequency of the $n$th lateral Fourier mode (lower index of the mode amplitudes) is $n \omega$ with $\omega$ being the basic frequency of the TW. With the complex vector of TW mode amplitudes

$$
\widehat{\mathbf{X}}_{\mathrm{TW}}=\left[\widehat{T}_{2 n}^{2(m+1)}, \widehat{T}_{2 n+1}^{2 m+1}, \widehat{\zeta}_{2 n}^{2 m+1}, \widehat{\zeta}_{2 n+1}^{2 m}\right]^{\mathrm{T}}
$$

the system of the projected balance equations reduces once more to a linear algebraic system

$$
\underline{\underline{\mathcal{M}}}_{\mathrm{TW}}\left(\omega, w_{\max }\right) \cdot \widehat{\mathbf{X}}_{\mathrm{TW}}=\mathbf{B}_{\mathrm{TW}}\left(w_{\max }\right)
$$

where, however, the matrix $\underline{\underline{\underline{\mathcal{M}}}} \mathrm{Tw}$ of mode coupling coefficients is complex. Another complex equation (or two real ones) generated by the momentum balance relates the two real groups $\omega$ and $w_{\max }$ with the Rayleigh number $R$ and the separation ratio $\psi$

$$
\left(\begin{array}{cc}
\mathbf{a}_{11} \widehat{\mathbf{X}}_{\mathrm{TW}} & \mathbf{a}_{12} \cdot \widehat{\mathbf{X}}_{\mathrm{TW}} \\
\mathbf{a}_{21} \cdot \widehat{\mathbf{X}}_{\mathrm{TW}} & \mathbf{a}_{22} \cdot \widehat{\mathbf{X}}_{\mathrm{TW}}
\end{array}\right)\left(\begin{array}{c}
1 / R \\
\psi
\end{array}\right)=\left(\begin{array}{c}
\mathbf{b}_{1} \cdot \widehat{\mathbf{X}}_{\mathrm{TW}} \\
\mathbf{b}_{2} \cdot \widehat{\mathbf{X}}_{\mathrm{TW}}
\end{array}\right)
$$

with $\mathbf{a}_{i k}, \mathbf{b}_{i}$ being vectors of projection coefficients.

A possibility to solve the system is to solve (3.11) for a given combination $\left(\omega, w_{\max }\right)$ and to use the resulting $\widehat{\mathbf{X}}_{\mathrm{TW}}\left(\omega, w_{\max }\right)$ for solving 3.12$)$ with resepect to $(1 / R, \psi)$. This means that $R$ and $\psi$ are uniquely determined for a given combination $\left(\omega^{2}, w_{\max }^{2}\right)$. The relevant parameters are here the squares $\omega^{2}$ and $w_{\max }^{2}$ since left- and right-traveling waves are symmetry degenerated and the convective amplitude $w_{\max }$ was chosen to be positive by (3.1).

This result is illustrated in Fig. 3: The TW states fill the $\omega^{2}-w_{\max }^{2}-$ plane. The lines are connecting states along a TW bifurcation branch for a particular separation ratio. The 
control parameter $R$ varies along a particular line between $R_{\text {osc }}$ at the Hopf bifurcation $\left(w_{\max }=0\right)$ and $R^{*}$ at the SOC-TW-transition $\left(\omega^{2}=0\right)$ in a way that can be nonmonotonous. The value of $\omega^{2}$ for $w_{\max }^{2}=0$ is $\omega_{H}^{2}$, i. e., the square of the Hopf frequency $\omega_{H}$. The dotted line represents the position of the saddle nodes in the diagrams $\omega(R)$ or $w_{\max }(R)$. It vanishes in the vicinity of $\psi=-0.01$ since for weaker Soret couplings only an unstable lower TW bifurcation branch exists without any saddle node. These topological features will be discussed later in this article.

\section{Comparison with finite difference numerical results}

In Fig. 1 we give a quantitative comparison of bifurcation diagrams computed by the above presented numerical Galerkin method and those obtained by a finite difference MAC scheme with a full representation of the fields. In our Galerkin scheme, we used one velocity mode and up to 19 temperature and $\zeta$ field modes in each direction. So, we computed 761 real mode amplitudes in the case of TW convection.

For both SOC and TW states the pairs of diagrams coincide. The most evident dis-

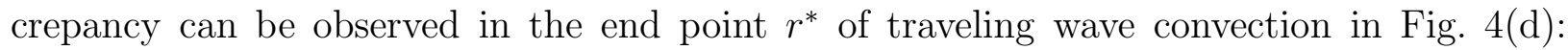
The MAC results with a discretization of $d x=d z=0.05$ (solid circles) predict $r^{*} \simeq 1.65$ [16], the Galerkin scheme (dashed line) $r^{*} \simeq 1.495$. In order to elucidate this deviation we performed a finite difference calculation with $d x=d z=0.025$ (open lozenges) and observed $r^{*} \simeq 1.45$, i. e., close to the value of the Galerkin scheme which used modes up to a wave number $19 \pi$ in both directions. The variation of $r^{*}$ with the spatial resolution of the MAC scheme is caused by concentration boundary layers at the plates: Galerkin method and the finer resolving MAC scheme show the same thickness which is smaller than that predicted by the worse resolving method $d x=d z=0.05$. Now, the SOC-TW-transition may be interpreted as a boundary layer instability [28] that occurs if the SOC boundary layer thickness $\propto\left(L / w_{\max }\right)^{1 / 3}$ exceeds a certain value when reducing $r$ or reducing the convective amplitude. This critical thickness is reached for higher amplitudes when using a numerical 
method that produces larger boundary layers. In so far, $r^{*} \simeq 1.45$ is a more adequate value for $L=0.01, \sigma=10$, and $\psi=-0.25$ than $r^{*} \simeq 1.65$ [16].

The deviations in the bifurcation diagrams for the variance $M$ of the concentration in Fig. $4(b, e)$ are mainly due to the shift in the frequency bifurcation branches. This means that the dependence of the concentration distribution on the frequency is reproduced well.

The convective heat transport $N-1$ [Fig. $4(\mathrm{c}, \mathrm{f})]$ in our Galerkin approximation is carried by only one velocity mode so that the actual values may be expected to be larger the higher the forcing, i .e., the Rayleigh number $r$. This typical behaviour can be observed also for the pure fluid $\psi=0$. There, a discrepancy between full MAC results and our Galerkin approximation of about $6 \%$ at $r=2$ is seen.

\section{RESULTS}

\section{A. Soret coupling and bifurcation topology}

The dependence of the bifurcation topology on the strength of the Soret coupling, say in the range $-0.25<\psi<0$, was not yet discussed in the literature [14, 116,28] in detail: On the one hand, the directly integrating numerical methods [14,16 require large amounts of CPU time due to critical slowing down near the saddle node positions $r_{\mathrm{SOC}}^{s}$ and $r_{\mathrm{TW}}^{s}$. On the other hand, the Soret effect was only implemented incompletely in a theoretical approach [28]. The same applies to the transition point $r^{*}$ of TW to stationary convection. The Galerkin method presented in the last section does not suffer from these disadvantages and it yields also the whole unstable branches directly without any numerical tricks. Since it computes only fixed points the intrinsic time scale of the system does not enter the problem. However, an additional stability analysis of the computed states is necessary. 


\section{Strong negative $\psi$}

The investigation of the variation of the bifurcation scenario with the separation ratio $\psi$ has shown for strong negative couplings a very interesting feature [17]: the development of a lower stable TW branch out of the bump seen in Fig. 1 in the unstable branch for $\psi=-0.25$. For $\psi \lesssim-0.4$ two stable, convective TW solutions exist opening up the possibility of the coexistence of two different traveling states in one container. Furthermore, all these TWs were found to display universal scaling properties [17]: (i) The mixing $M$, i. e., the concentration variance varies linearly with the TW frequency. (ii) The latter itself is uniquely fixed by the "distance", $r\left(w_{\max }, \psi\right)-r\left(w_{\max }, \psi=0\right)$, of the TW state's location $\left(r, w_{\max }\right)$ on the TW bifurcation branch from the pure fluid $(\psi=0)$ convection coordinates in the $r-w_{\max }$ bifurcation diagram. Thus, bifurcation properties, spatial structure of the concentration distribution, and TW dynamics show for strong Soret coupling a peculiar scaling behaviour. For a discussion of the characteristic changes in the bifurcation topology at strong Soret coupling see Ref. [17].

\section{Weak negative $\psi$}

On the side of weak negative Soret couplings, i. e., $\psi \rightarrow 0$, the motion of the saddle nodes $r_{\mathrm{SOC}}^{\mathrm{s}}$ and $r_{\mathrm{TW}}^{\mathrm{s}}$ and of the $\mathrm{SOC}-\mathrm{TW}$ merging point $r^{*}$ in the $r-\psi$ plane was not elucidated except for the vanishing of stable TW convection for $\psi>-0.01$ in mixtures with ethanolwater parameters $L=0.01$ and $\sigma=10$ [16]. To fill this gap we discuss in Figs. 010 the bifurcation properties of TWs and SOCs in the range $-0.25<\psi<0$. In Fig. 5 we show TW and SOC bifurcation diagrams of $w_{\max }^{2} v s r$ for several $\psi$ as indicated. In the case of the strongest coupling $\psi=-0.05$ [Fig. [5(a)] the same situation as for $\psi=-0.25$ (Fig. 1) is observed except for the fact that here $r^{*}<r_{\text {osc }}$ so that a SOC state is observed when heating above threshold. The stationary bifurcation threshold, $r_{\text {stat }}$, is negative as it is the

case for all $\psi<\frac{-L}{1+L}=-\frac{1}{101}$ for $L=0.01$ [20.19]: Thus, the SOC branch is disconnected 
with the ground state $w_{\max }^{2} \equiv 0$ at the positive $r$-axis. The shape of the SOC branch does not change when reducing the strength of the Soret effect because the tricritical separation ratio for SOCs, $\psi_{\text {SOC }}^{t}$, scales with $-L^{3}$ 29] and is effectively 0 for small Lewis numbers. The most evident effect is the motion of the SOC-TW-transition point $r^{*}$ along the SOC branch towards the SOC saddle node. For $\psi=-0.02$ [Fig. 5(b)], the TW and SOC saddle nodes coincide; for $\psi=-0.01$ [Fig. 5(c)] the TW branch merges with the SOC branch at the SOC saddle node. The transition from SOC to a pair of symmetry degenerated TWs at $r^{*}$ which is a pitch fork bifurcation of the TW frequency is still backwards in the sense that the frequency bifurcation is subcritical. Different to $\psi=-0.02$ we can observe for $\psi=-0.01$ TWs also for control parameters smaller than those of all SOCs, i. e., the extended state with the smallest Rayleigh number is now a TW and no longer a SOC. This is different from the behaviour for $\psi<-0.02$ where SOCs exist also at Rayleigh numbers not allowing TWs.

In Fig. 5(d) with $\psi=-0.0085$ the TW branch merges with the lower, unstable SOC branch which then becomes stable beyond its saddle node. But the TW saddle is still found at smaller amplitudes and Rayleigh numbers than that of the SOCs. The consequences are as follows: The lower TW branch locates unstable TWs which become stable at the saddle. However, they change stability once more since stable TWs may not merge with the unstable SOC branch. The only possibility is the existence of an additional bifurcation point — here to a modulated TW (MTW) - on the upper TW branch between $r_{\mathrm{TW}}^{\mathrm{s}}$ and $r^{*}$. This scenario is investigated in more detail in the next subsection IVA3.

Here, we continue the discussion of the changes in the bifurcation topology of ethanolwater mixtures for $\psi \rightarrow 0$. At $\psi=-0.007$ in Fig. 5(e) the TW saddle has vanished and the whole TW branch is unstable. The next significant change in the topology occurs at the tricritical separation ratio $\psi_{\mathrm{TW}}^{t} \simeq-5 \cdot 10^{-5}$ [29]. Therefore, at $\psi=-4 \cdot 10^{-5}$ we observe in Fig. $5(\mathrm{f})$ a supercritically bifurcating TW branch. It is stable just at the onset and then becomes unstable with respect to MTWs. At the codimension-2-point $\psi_{\mathrm{CT}}=-3.526 \cdot 10^{-5}$ 29] the TW branch vanishes completely. For separation ratios $\psi$ between $\psi_{\mathrm{TW}}^{t}$ and $\psi_{\mathrm{CT}}$ there is also a necessity of a change in stability along the supercritically bifurcating TW 
branch ending on the unstable SOC branch.

\section{Modulated TWs}

We have checked the scenario for the appearence of MTWs more explicitly for a parameter combination which is realized in ${ }^{3} \mathrm{He}-{ }^{4} \mathrm{He}$-mixtures rather than in ethanol-water, namely $L=0.03, \sigma=1$, and $\psi=-0.055$. In ${ }^{3} \mathrm{He}-{ }^{4} \mathrm{He}$ this scenario occurs in a four times broader range of the control parameter $(r \in[1.0612,1.0614]$, see Fig. 6(a) $)$ than it is realized in ethanol-water mixtures $(r \in[1.01375,1.01380]$, see Fig. 国(d)).

Sufficiently above the oscillatory threshold $r_{\text {osc }}=1.08827\left(\omega_{H}=3.8467, k_{c}=3.1152\right)$ stable SOCs (filled triangles) can be realized according to Fig. 6(a). When reducing the heating rate $r$ we approach the $\mathrm{SOC}-$ saddle at about $r_{\mathrm{SOC}}^{s} \simeq 1.06139$. Below that, the system falls down on the branch of TWs which is oscillatory unstable (open squares) at $r_{\mathrm{SOC}}^{s}$. The upper stable TW branch (filled circles) turns unstable at $r_{\mathrm{MTW}} \simeq 1.06125$ shortly above the saddle $r_{\mathrm{TW}}^{s}$. The oscillatory unstable TWs (open squares) get modulated by a frequency at least ten times smaller than the basic frequency of the TW itself - compare the frequencies in Fig. 6(b) and the imaginary parts of the relevant eigenvalues in Fig. E(c). The instability of the TWs on their upper branch, i. e., the transition at $r_{\mathrm{MTW}}$ from filled circles to open squares, gives birth to MTWs with slowly breathing amplitude. The type of the instability of the TWs changes on the upper TW branch at $r \simeq 1.06141$ from oscillatory to stationary (open circles) in order to merge appropriately with the stationary unstable lower SOC branch (open triangles).

This scenario has also been discussed in a minimal model by Knobloch and Moore [30]. They, however, investigated a system with stress free and, more importantly, permeable boundaries without adequately resolving the boundary layers. We give here evidence for MTWs in binary mixtures with realistic boundary conditions realized in the experiments. Note, however, the experimental investigation requires a control of the temperature difference of about $10^{-4}$. 


\section{Phase diagram}

Our results are summarized in a phase diagram in Fig. 7 where the $\psi$-dependence of $r^{*}$ (open lozenges), SOC saddle (open triangles up), and TW saddle (filled circles) are displayed together with the linear stability thresholds of the basic state (stationary: open triangles down, oscillatory: filled squares). Stable TW states on the upper TW bifurcation branch are located in the shaded region of Fig. [1. The inset in the upper right corner covers separation ratios between $\psi=-0.05$ and $\psi=-0.005$. In this inset we have scaled $r-1$ by $\sqrt{-\psi}$ in order to map the SOC saddle node position $r_{\mathrm{SOC}}^{s}$ approximately onto a constant since its $\psi$-dependence may be fitted very well by

$$
r_{\mathrm{SOC}}^{s} \simeq 1+1.636 \sqrt{-L \psi}
$$

However, in view of the fact that the exponent in (4.1) is not exactly $1 / 2$ it is not surprising that $r_{\mathrm{SOC}}^{s}$ is not exactly constant in the inset of Fig. 7 . The range of existence of the MTWs is too small to be visible in Fig. Q.

The changes of the bifurcation topology induced by increasing the strength of the negative Soret coupling beyond values of -0.25 including a detailed phase diagram for $\psi \in[-0.7,-0.2]$ were discussed in Ref. [17]: There, two stable, nonlinear TW branches have been discovered for $\psi \lesssim-0.4$ in mixtures with $L=O(0.01)$ and $\sigma=O(10)$.

\section{B. Concentration distribution and streamfunction}

Fig. 8 shows a combined bifurcation diagram of convective amplitude $w_{\max }$ and phase velocity $v$ vs. reduced Rayleigh number $r$. The TW states have been computed with our Galerkin method using the one-mode velocity field approximation (3.1). Four states are labelled by A to D in agreement with Fig. 1. 1 .

We want to discuss here the changes in the concentration distribution when moving along the TW bifurcation branch and their relation to the structural changes of the streamfunction 


$$
\tilde{\phi}(x, z)=\frac{w_{\max }}{k \mathcal{C}_{1}(0)} \sin k x \mathcal{C}_{1}(z)+v z
$$

for the velocity field (3.1) in the frame that is comoving with the TW phase velocity $v$. Note that in this frame the velocity field is stationary so that passive particles would move along the streamlines of 4.2 ).

The first occurrence of local extrema in $\tilde{\phi}(x, z)(4.2)$ gives rise to the first appearence of areas of closed streamlines. That happens for $k=\pi$ at the velocity ratio $\chi=\frac{w_{\max }}{v}=0.9807$. Thus, for $\chi \lesssim 1$, i. e., in all TW states between the Hopf bifurcation threshold $r_{\text {osc }}$ and state A the streamlines are all open. Increasing the convective amplitude $w_{\text {max }}$ yields decreasing phase velocity $v$ and increasing values of $\chi$ so that areas of closed streamlines appear that grow on cost of those occupied by open streamlines. For $v=0$, i. e., at the point $\mathrm{D}$ only closed streamlines are observed.

Without feedback into buoyancy concentration is a passive scalar transported by means of convection and diffusion (for the discussion of the Soret coupling in the bulk equations see Sec. [VQ). Then one can apply the results of passive scalar theory, e. g., [31, 32]. It explains that within closed streamlines a weakly diffusing scalar is homogenized. This behaviour is elucidated in Fig. 9 where we show lateral and vertical concentration profiles for the states A - D labelled in Fig. 8. In the states B, C, and D where $\chi>1$ and therefore areas of open streamlines exist we see a plateau characteristic of both profiles. However, the state A with only open streamlines shows no such plateaus.

Note that the lateral concentration wave profiles between the Hopf bifurcation and the state A are basically harmonic and that their amplitudes increase with increasing convective amplitude. This amplitude growth of the harmonic concentration wave occurs as long as there are not yet closed streamlines, i. e., up to a limiting point in the vicinity of state A. The amplitude of the wave profile of the state B in Fig. 9 is larger than that of state A as the lateral profiles are not taken at the center of the closed streamlines but in the center of the convection cell. Beyond this maximum amplitude the concentration wave crests are "cut off" and a plateau develops with the appearence of closed streamlines. The plateau 
extension, i .e., the region of constant concentration within the areas of closed streamlines broadens with increasing $\chi$ while simultaneously the plateau value of the concentration wave reduces. Furthermore, a small concentration peak at the leading front of the right traveling wave develops. It is caused by the advective injection of the concentration at the plates into the areas of closed streamlines. The lateral profile of state A has only a very weak, not visible asymmetry with respect to reflection at $x= \pm 1 / 2$ whereas the asymmetry in state $\mathrm{B}$ is obvious and gets more and more pronounced in $\mathrm{C}$ and $\mathrm{D}$. The adevctive injection of concentration into the regions of closed streamlines takes — with diffusion being small compared to advection — the form of an inwards spiralling concentration jet that can be seen in Fig.3 of Ref. [16.

This strong relation between streamlines and concentration distribution is also demonstrated in Fig. 10 where we have plotted the concentration field $C$ vs. the streamfunction $\tilde{\phi}$ in the frame comoving with the TW state traveling to the right. Just at the Hopf bifurcation threshold the streamfunction in the comoving frame is $\tilde{\phi}=v z=\omega_{H} z / k$ reflecting a vanishing convective velocity field in the laboratory frame. Via the conductive concentration profile $C_{\text {cond }}=-\psi z$ we get the relation $C_{\text {cond }}(\tilde{\phi})=-\frac{k \psi}{\omega_{H}} \tilde{\phi} \simeq 0.070 \tilde{\phi}$ (dotted lines in Fig. 10) between concentration and streamfunction with $\tilde{\phi}$ varying between $-v / 2$ and $v / 2$, i. e., between -1.78 and 1.78 . In state $\mathrm{A}$, the conductive concentration distribution is slightly deformed and the streamfunction varies between -1.4 and 1.4 which are the values at the top and bottom plates. At these boundaries the concentration is in contrast to the streamfunction not constant so that a vertical shape of $C(\tilde{\phi})$ is observed there. In state B there are already small areas of closed streamlines with equilibrated concentration. In these regions $C(\tilde{\phi})$ is a constant. Moving along the TW bifurcation branch the areas of these regions keep on increasing via state $\mathrm{C}$ to state $\mathrm{D}$ which is a SOC state. In this state we observe a remarkable concentration variation around its mean value 0 only in a small streamfunction interval around 0, the separatrix in this state. This is a strong boundary layer phenomenon caused by the smallness of Lewis number $L=O(0.01)$ in comparison with the convective amplitude $w_{\max }=O(10)$. 


\section{Soret coupling and concentration current}

\section{Comoving frame of reference}

The impact of the Soret coupling on the concentration current in a TW is best understood by studying the current in a frame comoving with the TW's phase velocity $v$. In this frame, the velocity field $\tilde{\mathbf{u}}(x, z)=\left(-\partial_{z} \tilde{\phi}, 0, \partial_{x} \tilde{\phi}\right)$ is well approximated by the ansatz (4.2) according

to ansatz (3.1) and the discussion of Fig. 2. The corresponding streamlines can be seen in Fig. 11(a) for a TW state with $v=4 / \pi$, i. e., $\omega=4$.

In the comoving frame the concentration current $\tilde{\mathbf{J}}$ is given by

$$
\tilde{\mathbf{J}}=\tilde{\mathbf{u}} C-L \nabla(C-\psi T)
$$

In a relaxed TW state the relation

$$
\partial_{t} C=0=-\nabla \cdot \tilde{\mathbf{J}}
$$

holds so that $\tilde{\mathbf{J}}$ is divergence free. It is shown as a vector field plot in Fig. 11(a) in the left column together with the streamlines of the velocity field $\tilde{\mathbf{u}}$ for a TW propagating to the right. The structure of the current can be explained as follows: In the closed streamlines of the right rolls of Fig. 11 concentration is homogeneized to a level close to that of the upper plate. This is due to the vicinity of the right roll to this plate where the higher concentration $(C>0)$ is observed for negative Soret couplings and heating from below. The left area of closed streamlines is located next to the lower plate and contains therefore the lower concentration $(C<0)$. The two different signs of concentration - the mean concentration is normalized to zero - are the reason for the concentration current $\tilde{\mathbf{J}}$ to rotate clockwise in both clockwise and counterclockwise rotating rolls. In the vicinity of the center open streamline that meanders between the rolls, a line of vanshing concentration current exists. It also vanishes near the center of the rolls where the velocity $\tilde{\mathbf{u}}$ vanishes.

At the plates the concentration current is purely lateral since due to the impermeable boundary condition $\tilde{\mathbf{J}} \cdot \mathbf{e}_{z}=0$. It is mainly given by $-v C$ being negative (positive) at 
the top (bottom) plate with $C>0(C<0)$. The contribution from lateral concentration gradients along the plates to the concentration current (4.3) is mutiplied by the Lewis number $L=O(0.01)$ and can therefrore be neglected there. The temperature, and consequently the Soret effect, does not contribute at all to $\tilde{\mathbf{J}} \cdot \mathbf{e}_{x}$ at the plates since the temperature is fixed there.

\section{Approximate Soret induced current}

Next, we discuss the influence of the temperature field on the concentration current (4.3) in the bulk. We do this by studying the left column of Fig. 11(b). There, $L \psi \nabla T$ is plotted by arrows whose lengths are magnified by a factor 30 relative to that of $\tilde{\mathbf{J}}$ in Fig. 11(a). For negative Soret coupling $L \psi \nabla T$ is parallel to the diffusive heat current $-\boldsymbol{\nabla} T$ pointing upwards in the system heated from below. The important thing is the existence of this current and its mean upwards direction and not the small modulations. We ignore these small lateral modulations by replacing $L \psi \nabla T$ by an adequate mean. For this mean we choose the mean of $L \psi \nabla T$ at the plates, namely

$$
\langle L \psi \nabla T\rangle_{x, z= \pm 1 / 2}=-L \psi N \mathbf{e}_{z}
$$

in order to guarantee the impermeability of the plates in the lateral mean. A global averaging of $L \psi \boldsymbol{\nabla} T$ would lead to $-L \psi \mathbf{e}_{z}$ differing from our choice only by a factor $N=O(1)$ and violating the impermeability by the same amount.

The replacement of $L \psi \nabla T$ by the mean (4.4) leads to the modified concentration current

$$
\tilde{\mathbf{J}}_{\text {modified }}=\tilde{\mathbf{u}} C-L \nabla C-L \psi N \mathbf{e}_{z}
$$

shown in the right column of Fig. 11(a). Therefore, in the modified concentration balance

$$
0=\partial_{t} C=-\nabla \cdot \tilde{\mathbf{J}}_{\text {modified }}=-\nabla \cdot[\tilde{\mathbf{u}} C-L \nabla C]
$$

the tempreature field has disappeared. It occurs only in the boundary condition

$$
0=\mathbf{e}_{z} \cdot \tilde{\mathbf{J}}_{\text {modified }}=-L \partial_{z} C-L \psi N \quad \text { at } z= \pm 1 / 2
$$


This allows the concentration field to be described by

$$
\begin{aligned}
C(x, z ; t)=-\psi N z & +\sum_{n=0}^{\infty} \sum_{m=0}^{\infty}\left[C_{2 n}^{2 m+1}(t) e^{-2 i n k x}+\text { c.c. }\right] \sqrt{2} \sin (2 m+1) \pi z \\
& +\sum_{n=0}^{\infty} \sum_{m=0}^{\infty}\left[C_{2 n+1}^{2 m}(t) e^{-i(2 n+1) k x}+\text { c.c. }\right] \sqrt{2} \cos 2 m \pi z
\end{aligned}
$$

Since now the actual concentration balance is solved and not the balance for the $\zeta$-field the above discussed problems with the relevance of temperature modes that are $1 / L$ times smaller than the $\zeta$-field modes do no longer occur. Consequently, the temperature field can now be represented by the simplest approximation

$$
\begin{aligned}
T(x, z, t)=-z & +T_{0}^{2}(t) \sqrt{2} \sin 2 \pi z \\
& +\left[T_{1}^{1}(t) e^{-i k x}+\text { c.c. }\right] \sqrt{2} \cos \pi z
\end{aligned}
$$

just like in the classical Lorenz model [33]. The advantage of this procedure is that only for the concentration field a many mode Galerkin representation is necessary and the temperature can be modelled by those modes which are observed in the fields and not additionally by those becoming necessary out of numerical reasons.

Bifurcation diagrams of the frequency computed by this approximate method (solid lines) are displayed in Fig. 12 and compared with the "exact" results (symbols) as described in Sec. III over a wide range of Soret couplings. We have chosen bifurcation diagrams for the frequency since it is most sensitive to an insufficient mode truncation. For separation ratios $\psi>-0.15$ no differences are observed whereas for stronger couplings $(\psi=-0.25)$ at high heating rates deviations become visible. The reason for this is the neglect of temperature modes higher than those incorporated in (4.7). At high heating rates they become relevant: The maximum of the second lateral temperature mode has reached for $r=1.6$ about $6 \%$ of the size of the first one (3\% for the third relative to the first) (see also [16, Fig.5b]). In so far, the simplest ansatz for the temperature field (4.7) cuts off at least $10 \%$ of the spectrum leading to comparable errors in the bifurcation diagram, especially in the vicinity of the SOC-TW-transition. 


\section{CONCLUSION}

We have given a detailed analysis of the influence of the Soret effect on thermal convection in binary liquid mixtures. As a tool we used a many mode Galerkin method combined with an approximation in the velocity field: It is truncated by a single mode which holds for convection in fluids with Prandtl numbers $\sigma \gtrsim 1$. The mean flow in traveling waves (TWs) was recognized as unimportant since it is very small and contributes only unsystematically to the main TW properties. This truncation of the velocity lead to a very simple and efficient solution procedure for the nonlinear TW and SOC fixed points by only solving linear equations. This opened up the possibility for a detailed elucidation of the changes in the combined bifurcation topology of stationary and traveling states, especially the existence range of TWs in the control and fluid parameter plane. Together with the investigation of strong Soret couplings [17 interesting fields for experiments are opened: the bistability of slow and fast traveling waves and the occurrence of modulated traveling waves with slowly breathing amplitude. The classification of the states is facilitated by a detailed phase diagram. As an additional insight it was found that the order paramters TW frequency and TW convective velocity determine the control parameters Rayleigh number and separation ratio uniquely.

Eliminating the intrinsic time scale from the computation of the fixed points allowed besides the determination of bifurcation points and saddle nodes the investigation of unstable states. In this regime the transition from weakly to strongly nonlinear TWs is observed and may be understood in the framework of the intimate relation between concentration distribution and the structure of the flow and the changes in this relation along the bifurcation branch.

As a further result the reason for the failure of earlier Galerkin approximations for the convection in binary liquid mixtures was revealed: In order to exactly fulfill the concentration boundary condition which is coupled to the temperature field earlier approaches used a combination of concentration and temperature field without resolving the temperature field 
adequately. When using this combined field for small Lewis numbers $L \ll 1$ a resolution of the temperature is required that goes beyond the dominating modes seen in simulations and experiments. Although the contribution of higher modes to the temperature field are small they are essential in the balance equations formulated with the combined field. A way out of this artificial theoretical dilemma is obtained by investigating the concentration current: It is mainly influenced by the lateral average of the temperature gradient in the system. This allows to ignore the Soret effect in the bulk equation of the concentration balance (bulk Soret effect) and to truncate the concentration field with an adequate boundary condition (boundary Soret effect) directly. Then, the foundations of a more compact description and solution for the convection in binary liquid mixtures are laid [34.

\section{ACKNOWLEDGMENTS}

This work was supported by the Deutsche Forschungsgemeinschaft. Stimulating discussions with W. Barten, P. Büchel, and H. W. Müller are gratefully acknowledged. 


\section{REFERENCES}

[1] M. C. Cross and P. C. Hohenberg. Rev. Mod. Phys. 65, 851 (1993).

[2] L. D. Landau and E. M. Lifshitz: Hydrodynamik, Akademie-Verlag, Berlin (1966)

[3] J. K. Platten and J. C. Legros, Convection in Liquids, Springer-Verlag, Berlin (1984).

[4] R. W. Walden, P. Kolodner, A. Passner, and C. M. Surko, Phys. Rev. Lett. 55, 496 (1985).

[5] H. Touiri, J. K. Platten, and G. Chavepeyer, Eur. J. Mech., B/Fluids, 15, No2, 241, (1996).

[6] G. Ahlers and I. Rehberg, Phys. Rev. Lett. 56, 1373 (1986).

[7] H. Gao and R. P. Behringer, Phys. Rev. A34, 697 (1986).

[8] E. Moses and V. Steinberg, Phys. Rev. Lett. 60, 2030 (1988).

[9] K. D. Eaton, D. R. Ohlsen, S. Y. Yamamoto, C. M. Surko, W. Barten, M. Lücke, M. Kamps, and P. Kolodner, Phys. Rev. A43, 7105 (1991).

[10] G. Zimmermann and U. Müller, Int. J. Heat Mass Transfer 35, 2245 (1992).

[11] B. L. Winkler and P. Kolodner, J. Fluid Mech. 240, 31 (1992).

[12] A. La Porta, K. D. Eaton, and C. M. Surko, Phys. Rev. E53, 570 (1996).

[13] W. Barten, M. Lücke, W. Hort, and M. Kamps, Phys. Rev. Lett. 63, 376 (1989).

[14] W. Barten, M. Lücke, and M. Kamps, in [15], p. 131.

[15] F. H. Busse and L. Kramer (Editors), Nonlinear Evolution of Spatio-Temporal Structures in Dissipative Continuous Systems, (NATO ASI Series B225, Plenum, New York, 1990).

[16] W. Barten, M. Lücke, M. Kamps, and R. Schmitz, Phys. Rev. E51, 5636 (1995).

[17] St. Hollinger, P. Büchel, and M. Lücke, Phys. Rev. Lett. 78, 235 (1997). 
[18] W. Hort, S. J. Linz, and M. Lücke, Phys. Rev. A45, 3737 (1992).

[19] St. Hollinger and M. Lücke, Phys. Rev. E52, 642 (1995).

[20] G. W. T. Lee, P. Lucas, and A. Tyler, J. Fluid Mech. 135, 235 (1983).

[21] S. J. Linz, M. Lücke, H. W. Müller, and J. Niederländer, Phys. Rev. A38, 5727 (1988).

[22] J. Niederländer, M. Lücke, and M. Kamps, Z. Phys. B82, 135 (1991).

[23] O. Lhost, S. J. Linz, and H. W. Müller, J. Phys. (Paris) II 1, 279 (1991).

[24] S. Chandrasekhar, Hydrodynamic and Hydromagnetic Stability, Dover Publications, New York (1981).

[25] S. J. Linz and M. Lücke, Phys. Rev. A35, 3997 (1987); Err., Phys. Rev. A36, 2486 (1988).

[26] H. Yahata, Prog. Theor. Phys., Supplement 99, 493 (1989).

[27] O. Lhost, Etude de la Convection Libre Induite par effet Soret, Thesis, Mons (Belgium) (1990, unpublished).

[28] D. Bensimon, A. Pumir, and B. I. Shraiman, J. Phys. (France) 50, 3089 (1989).

[29] W. Schöpf and W. Zimmermann, Phys. Rev. E47, 1739 (1993).

[30] E. Knobloch and D. R. Moore, in [15], p. 109.

[31] P. B. Rhines and W. R. Young, J. Fluid Mech. 133, 133 (1983).

[32] B. Shraiman, Phys. Rev. A36, 261 (1987).

[33] E. N. Lorenz, J. Atmospheric Sciences 20, 130 (1963).

[34] St. Hollinger, M. Lücke, and H. W. Müller, Phys. Rev. E57, 4250 (1998). 


\section{FIGURES}

FIG. 1. Bifurcation diagrams of convection flow intensity (a) and frequency (b) in TW (solid lines) and SOC (dashed lines) solutions in a binary mixture with $\psi=-0.25, L=0.01$ and $\sigma=10$. States A-D are identified for later discussion related to Figs. 8 -10.

FIG. 2. Quality of the one-mode velocity field approximation (3.1) for TWs with $L=0.01$, $\sigma=10$ and $\psi=-0.25$ (left column) and $\psi=-0.6$ (right column) as a function of growing flow amplitude $w_{\max }$ along the TW bifurcation branches. The top row displays the error in the vertical velocity field according to definition $(\overline{3.2})$ and the bottom row the maximal amplitude of the lateral mean flow.

FIG. 3. TW states in the $w_{\max }^{2}-\omega^{2}$-plane for $L=0.01$ and $\sigma=10$. The separation ratio $\psi$ is constant on each solid line. It varies logarithmically form line to line and has the value shown at the left ordinate. The dotted line gives the positions of the TW saddle nodes.

FIG. 4. Comparison of bifurcation diagrams of frequencies $\omega(\mathrm{a}, \mathrm{d})$, variance $M$ of the concentration field (b,e), and Nusselt number $N(\mathrm{c}, \mathrm{f})$ calculated by our Galerkin method and a finite difference MAC scheme. Results of the Galerkin method are shown by solid (SOC) and dashed (TW) lines. MAC results with a spatial resolution of $d x=d z=0.05$ [16] are shown by filled circles (TW), open squares (SOC), and open triangles (phase fixed, unstable SOCs). More accurate MAC results $(d x=d z=0.025)$ are displayed as open lozenges. The dotted lines show results of a pure fluid, $\psi=0$ : thick dots - Galerkin, thin dots - MAC.

FIG. 5. Bifurcation diagrams of $w_{\max }^{2}$ versus reduced Rayleigh number $r$ at weak Soret couplings and $L=0.01, \sigma=10$. Open lozenges represent SOCs, filled lozenges TWs. Results were computed with our Galerkin method. 
FIG. 6. Bifurcation diagrams of convective amplitude $w_{\max }$ (a) and frequency $\omega$ (b) for fluids with $L=0.03, \sigma=1$, and $\psi=-0.055$. Graph (c) shows eigenvalues determining the stability of the TWs: open (filled) symbols correspond to unstable, $\operatorname{Re} \gamma_{\mathrm{TW}}>0$, (stable, $\left.\operatorname{Re} \gamma_{\mathrm{TW}}<0\right)$ states (stationary: $\operatorname{Im} \gamma_{\mathrm{TW}}=0$, oscillatory: $\operatorname{Im} \gamma_{\mathrm{TW}} \neq 0$ ). The states corresponding to the small filled circles are not shown in (a) and (b).

FIG. 7. Phase diagram of the $\psi$-dependence in mixtures with $\mathrm{E}=0.01$ and $\sigma=10$ in a double logarithmical plot $r-1$ vs. $\psi$. SOC properties are shown by dotted lines (saddle node $r_{\text {SOC }}^{s}$ : open triangles up, bifurcation $r_{\text {stat }}$ : open triangles down). Solid lines correspond to points in the TW bifurcation diagrams (Hopf bifurcation $r_{\text {osc }}$ : filled squares, SOC-TW-transition $r^{*}$ : open lozenges, saddle node $r_{\mathrm{TW}}^{s}$ : filled circles).

FIG. 8. Bifurcation diagram of convective amplitude $w_{\max }$ and phase velocity $v$ vs. reduced Rayleigh number $r$ for TWs in a mixture with $L=0.01, \sigma=10, \psi=-0.25$, and $k=\pi$. The stable (unstable) branches are shown by solid (dotted for $w_{\max }$ and dashed for $v$ ) lines. Letters A - D identify states discussed in the text.

FIG. 9. Lateral and vertical concentration profiles of the four states identified in Figs. 1 and 8.

FIG. 10. Concentration $C$ vs. streamfunction $\tilde{\phi}$ in the comoving frame for the states identified in Figs. I and 8. The dotted lines referring to the conductive state are explained in the text. Since $C$ and $\tilde{\phi}$ was evaluated on a grid some of the fine structure seen in the plots reflects the grid discretization. 
FIG. 11. Concentration currents in a TW propagating to the right in the comoving frame ( $L=0.01, \sigma=10, \psi=-0.25$, and $\omega=4$ ) with complete Soret coupling (left column) and averaged, boundary Soret forcing (right column). For details see text. The top row (a) shows the total concentration current $\tilde{\mathbf{J}}(4.3)$ in the left column and the modified current $\tilde{\mathbf{J}}_{\text {modified }}(4.5)$ in the right column each together with the streamlines of $\tilde{\mathbf{u}}$. The scaling factor for the lengths of the arrows is 0.5 relative to the units of lateral and vertical axes. Row (b) shows the influence of the Soret coupling more explicitly. Left column: $L \psi \nabla T$ (complete coupling); right column: $-L \psi N \mathbf{e}_{z}$ (averaged coupling). In row (b), the arrow lengths had to be magnified by a factor 30 relative to those of the top row in order to make them visible.

FIG. 12. Bifurcation diagrams of TW frequencies. Symbols refer to correct Soret coupling and full mode field representation. Lines were obtained by the laterally averaged Soret effect (4.5) and the reduced temperature representation (4.7). The unstable states for the complete coupling have been dropped for reasons of clarity. Parameters are $L=0.01$ and $\sigma=10$. 


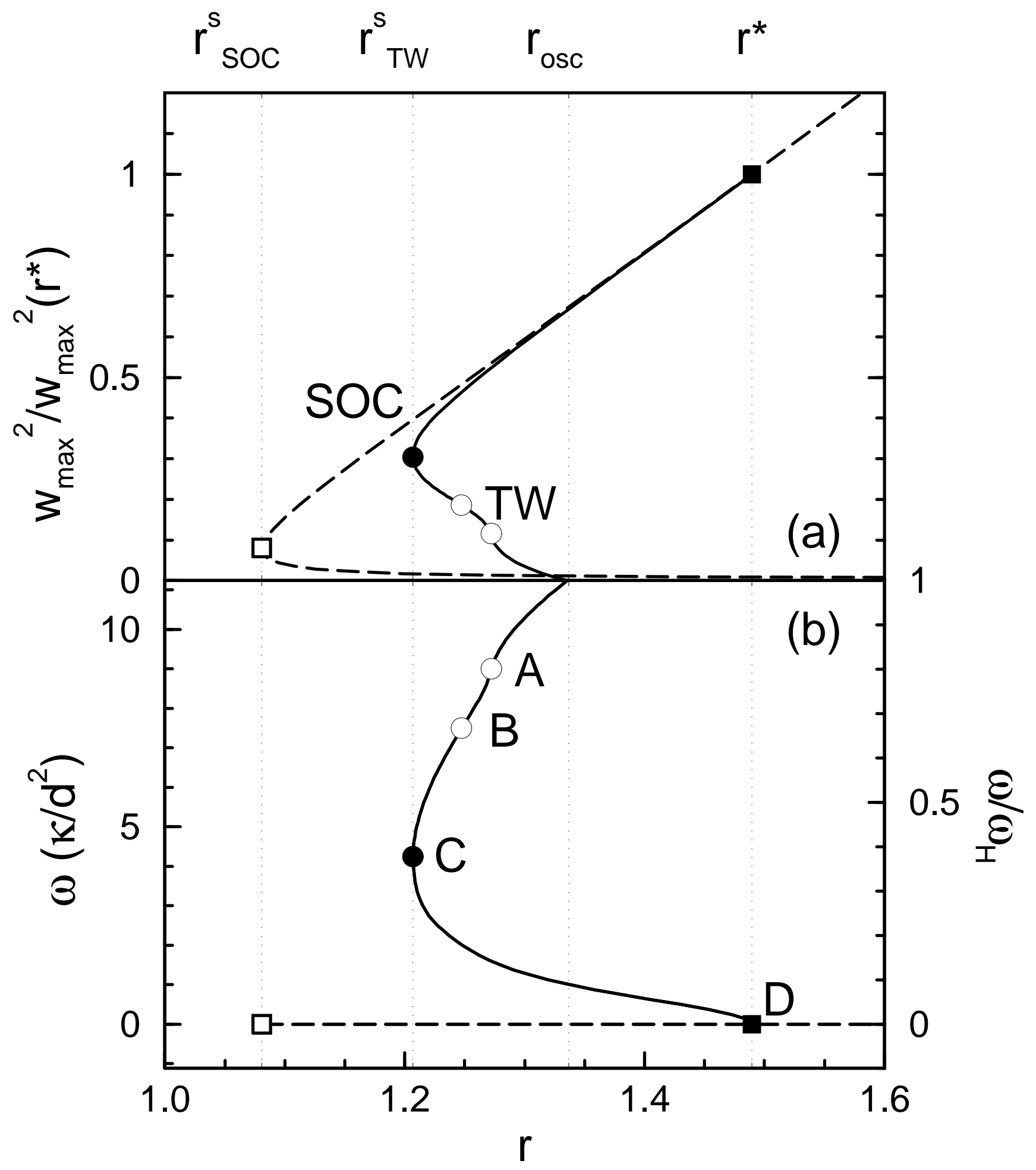

Fig. 1: Hollinger and Lücke, Phys. Rev. E

Influence of the Soret effect on convection of binary fluids 


$$
\psi=-0.25 \quad \psi=-0.6
$$

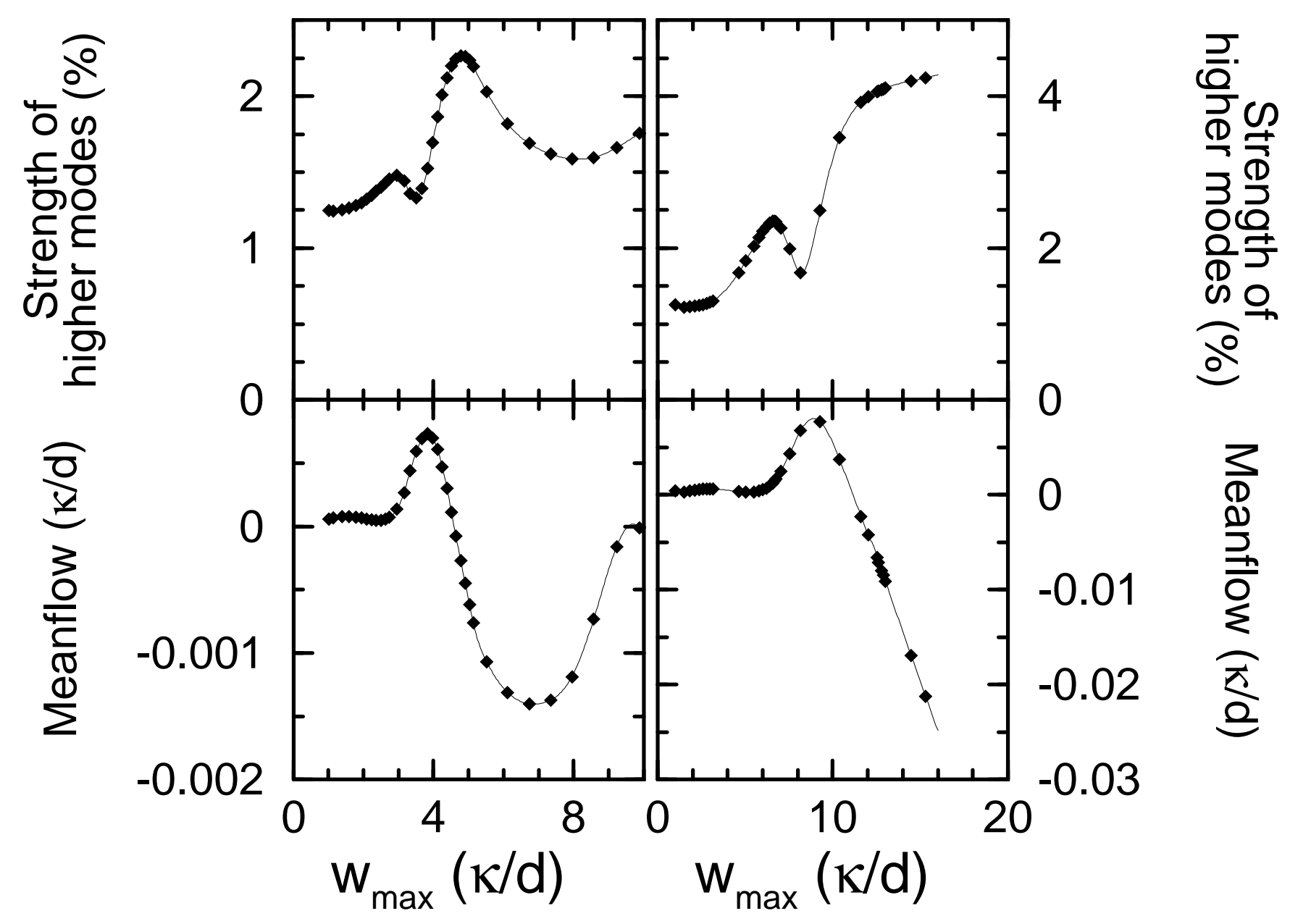

Fig. 2: Hollinger and Lücke, Phys. Rev. E Influence of the Soret effect on convection of binary fluids 


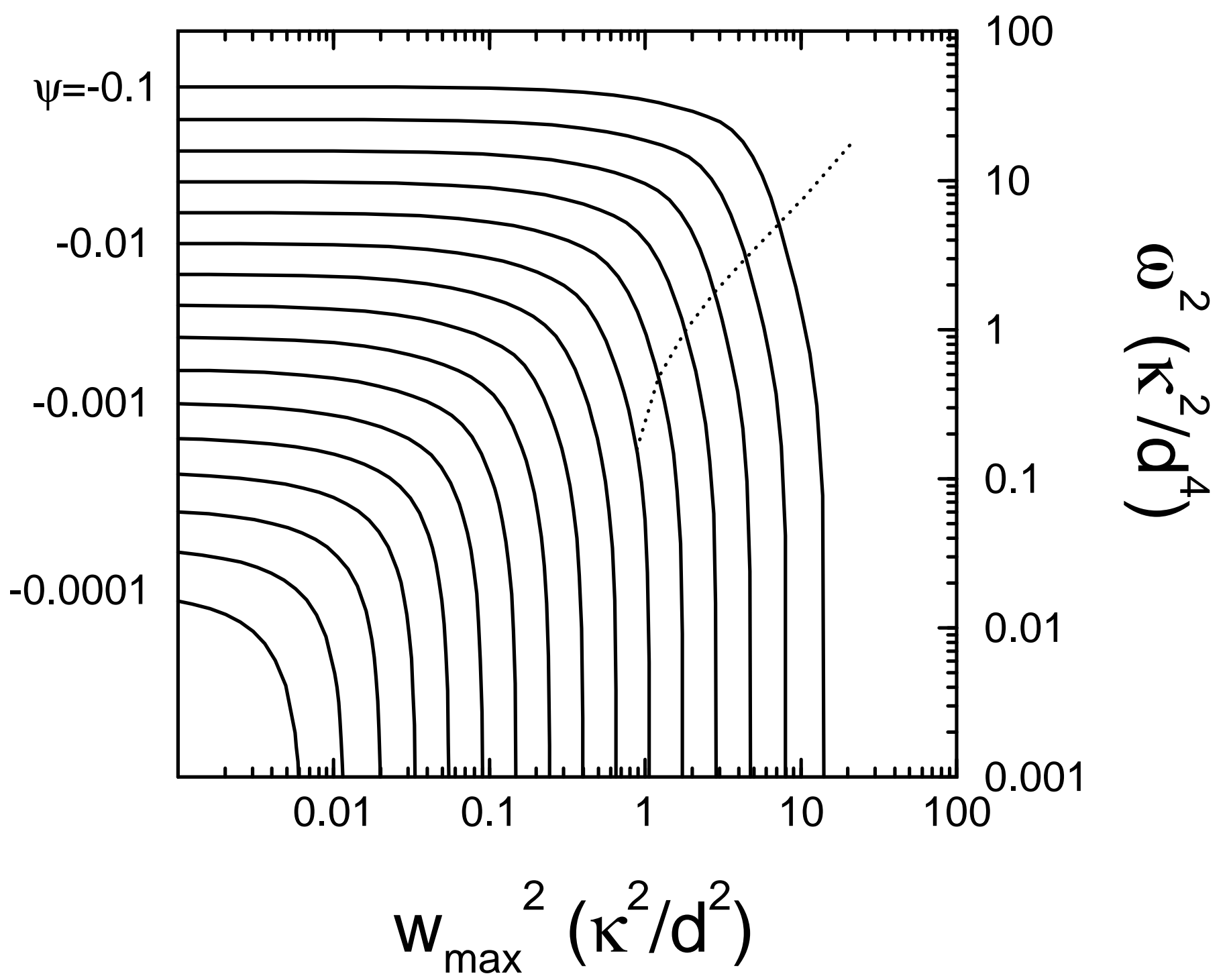

Fig. 3: $\quad$ Hollinger and Lücke, Phys. Rev. E Influence of the Soret effect on convection of binary fluids 


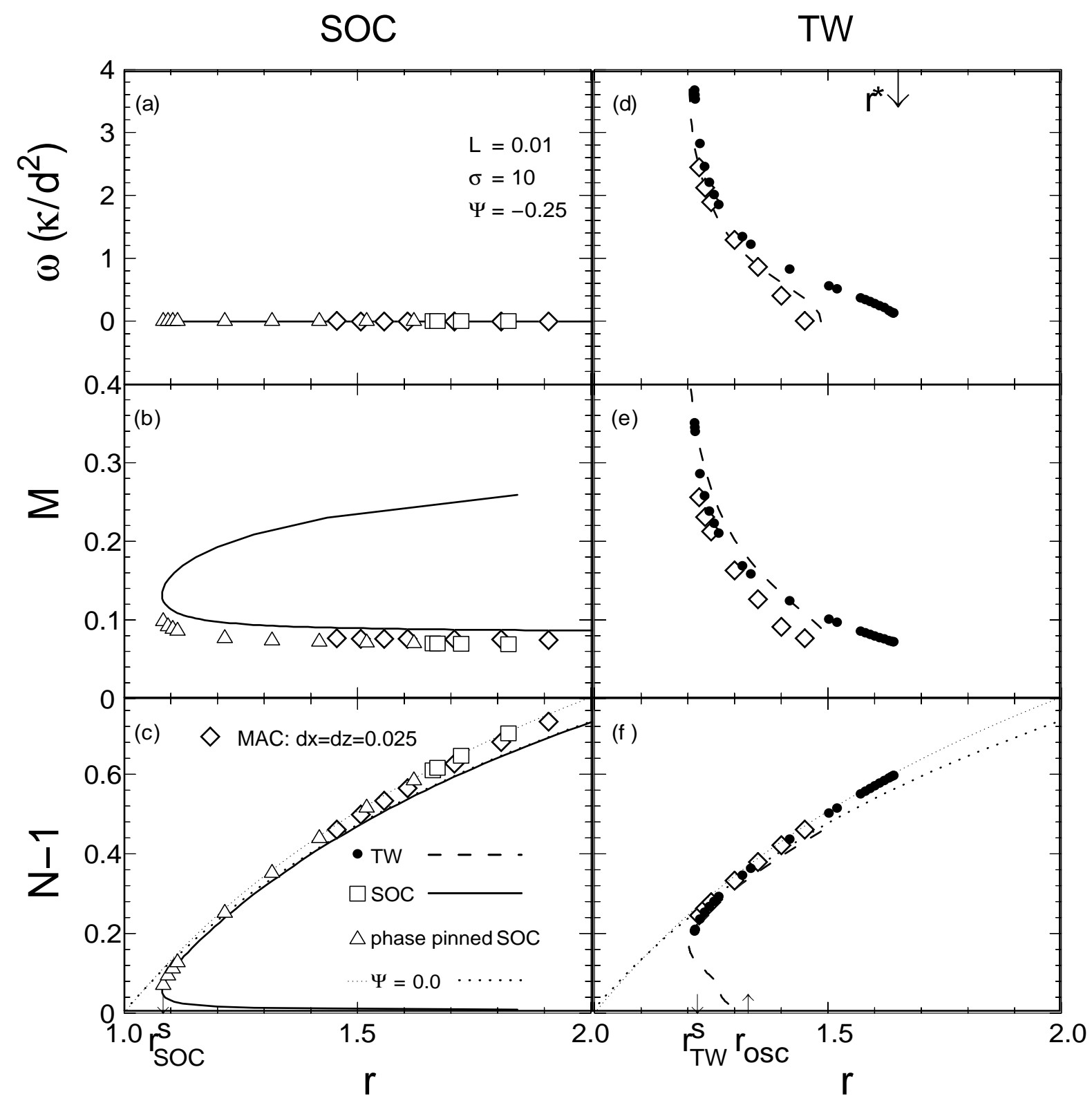

Fig. 4: Hollinger and Lücke, Phys. Rev. E Influence of the Soret effect on convection of binary fluids 


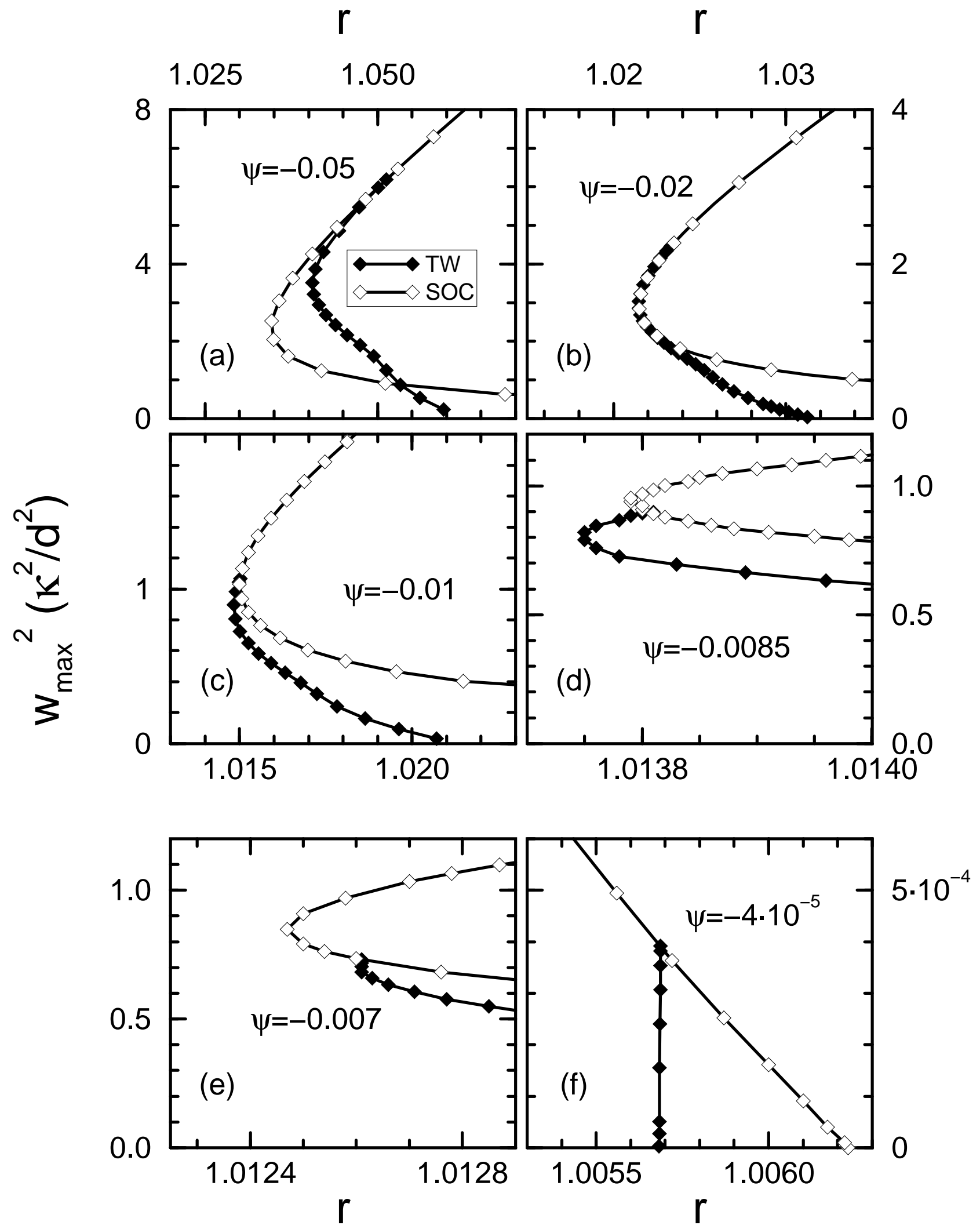

Fig. 5: Hollinger and Lücke, Phys. Rev. E Influence of the Soret effect on convection of binary fluids 


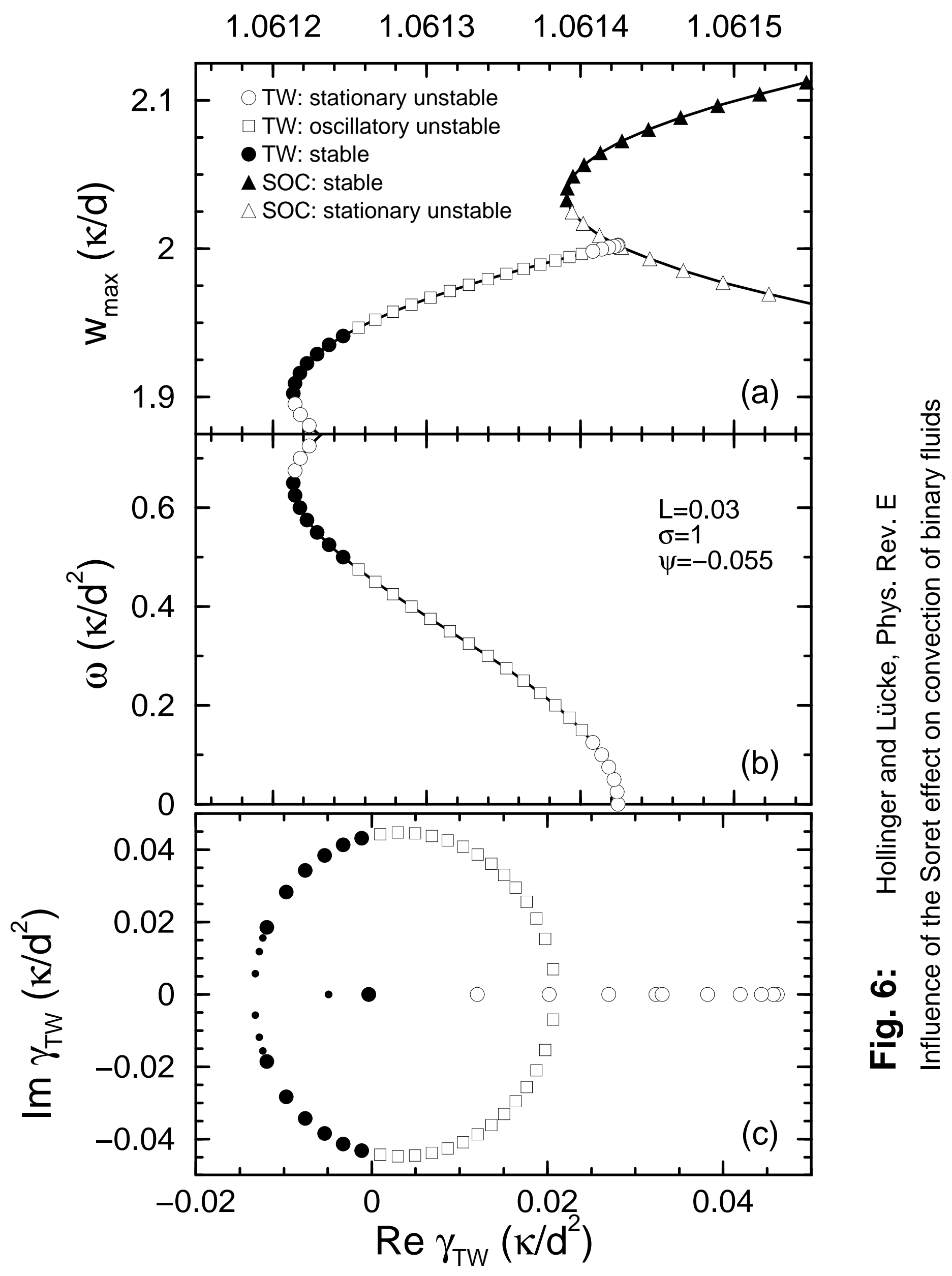




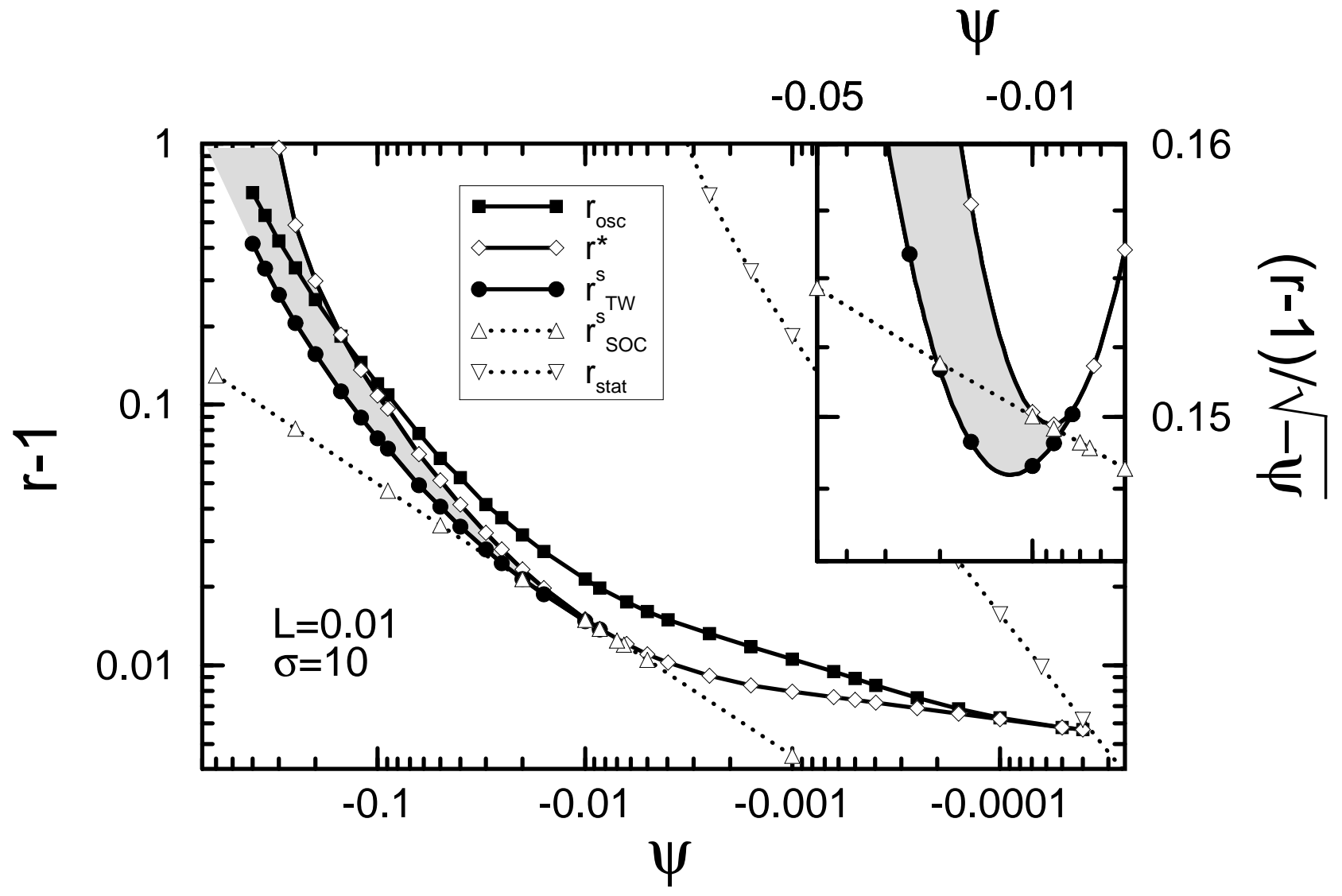

Fig. 7: $\quad$ Hollinger and Lücke, Phys. Rev. E Influence of the Soret effect on convection of binary fluids 
Fig. 8: Hollinger and Lücke, Phys. Rev. E Influence of the Soret effect on convection of binary fluids

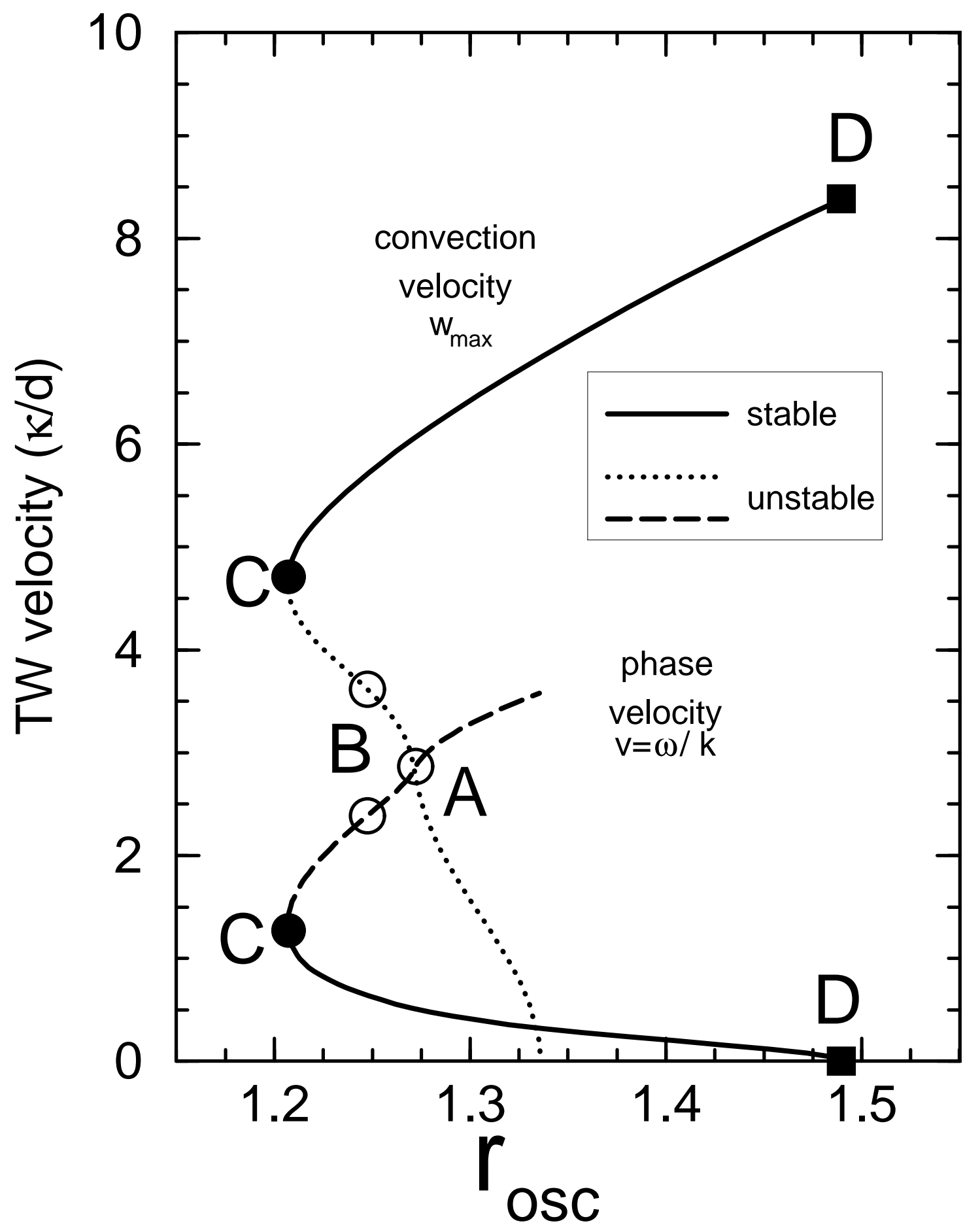

Rayleigh number $r$ 


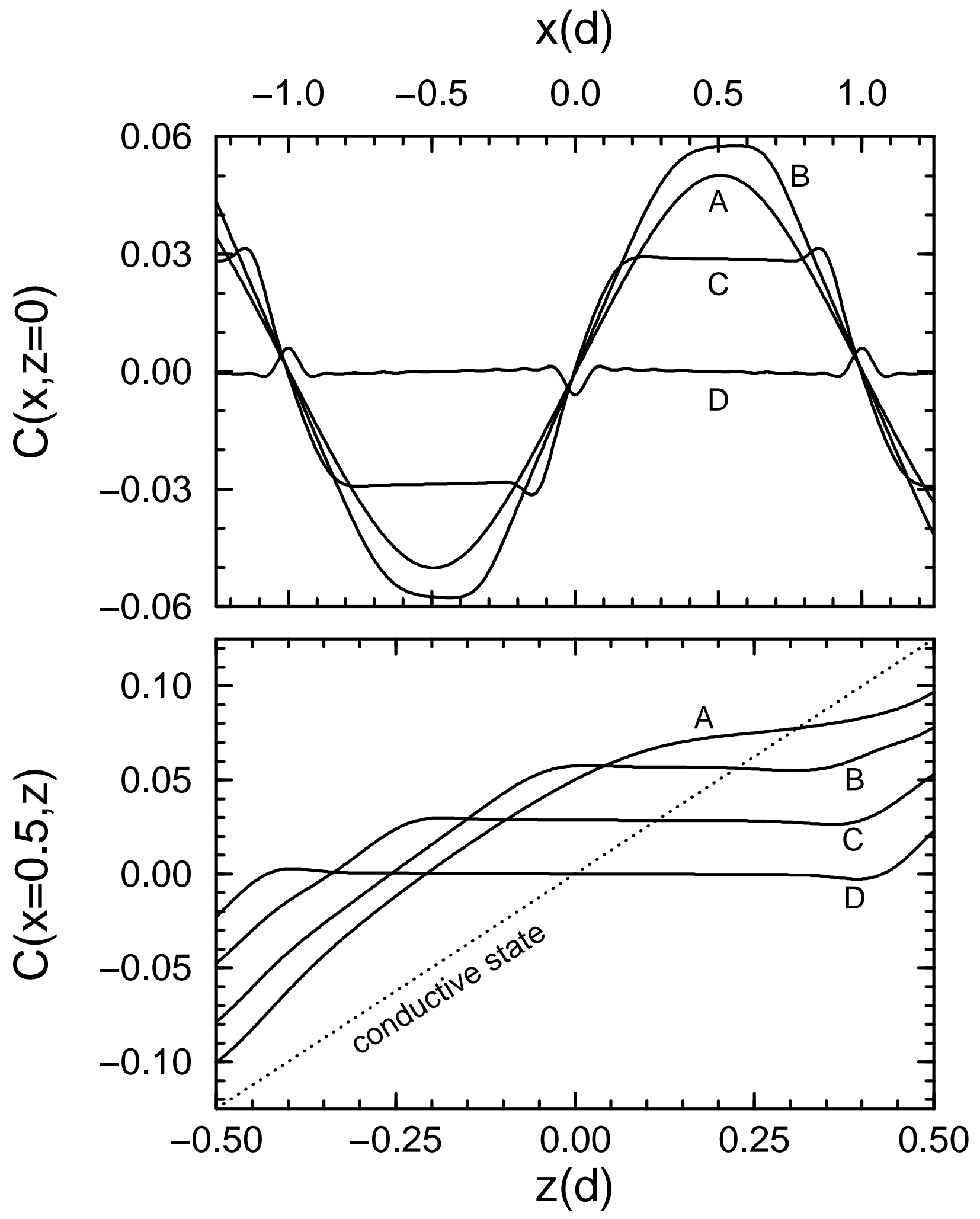

Fig. 9: Hollinger and Lücke, Phys. Rev. E Influence of the Soret effect on convection of binary fluids 


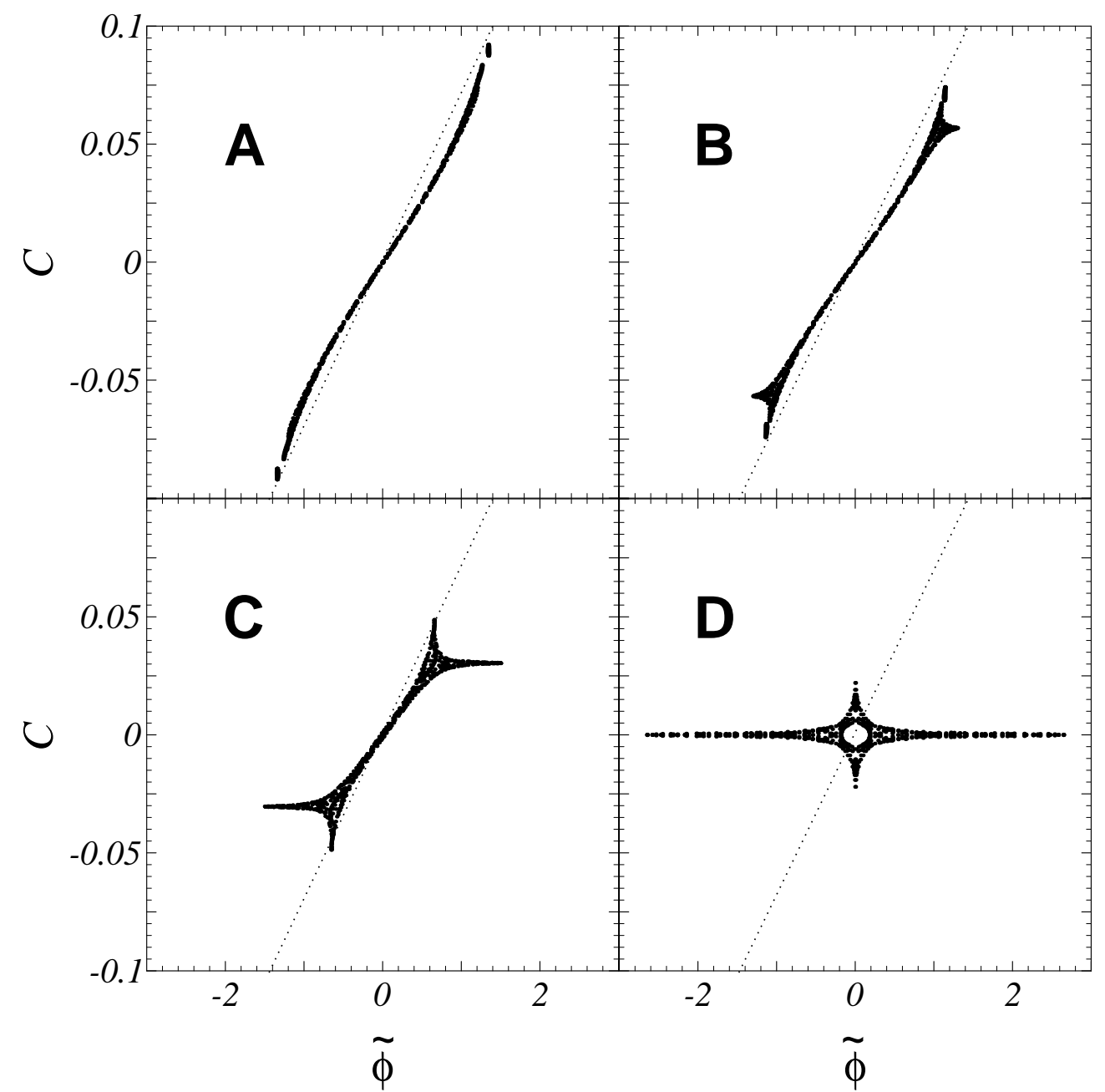

Fig. 10: Hollinger and Lücke, Phys. Rev. E Influence of the Soret effect on convection of binary fluids 


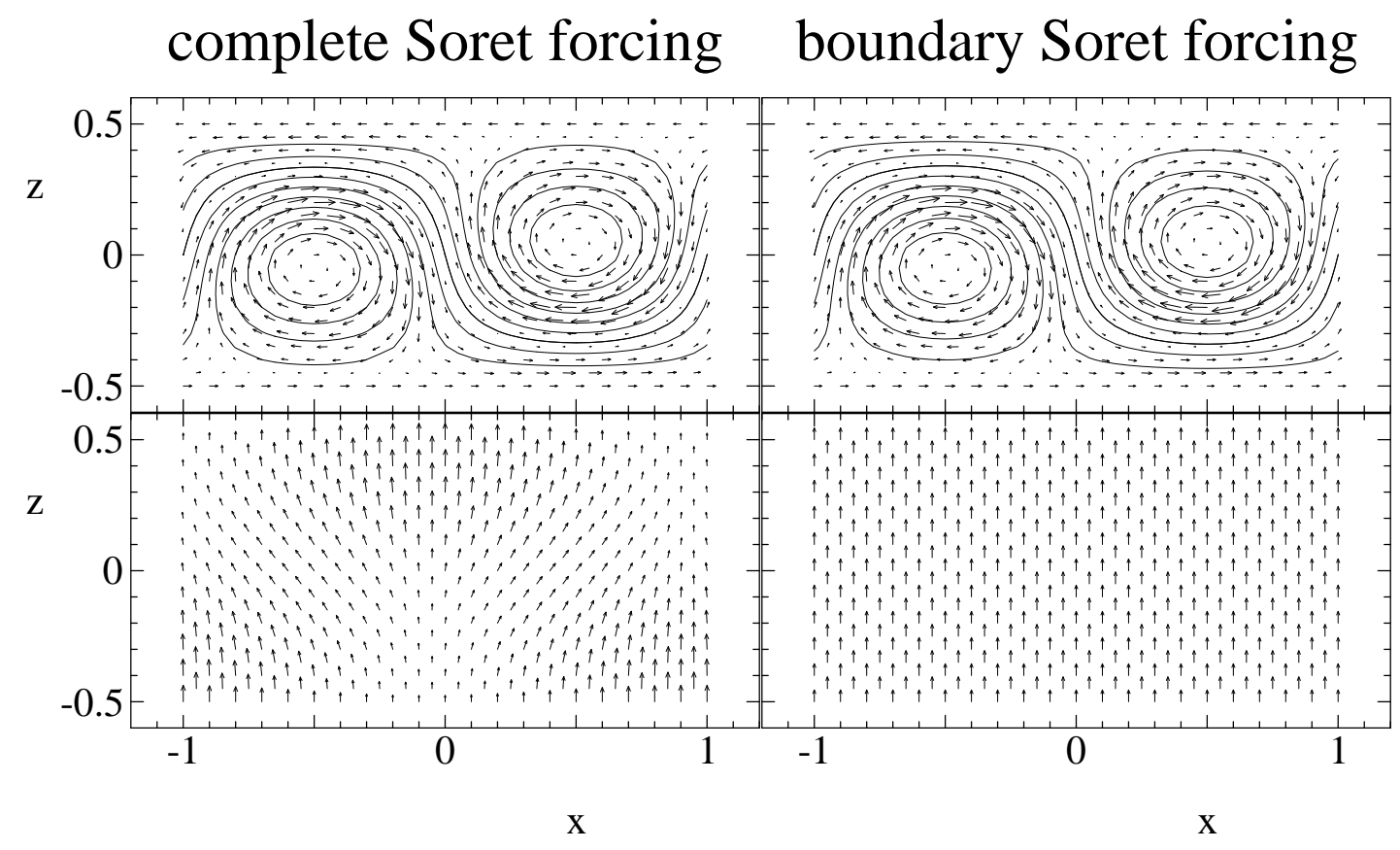

(a)

(b)

Fig. 11: Hollinger and Lücke, Phys. Rev. E Influence of the Soret effect on convection of binary fluids 


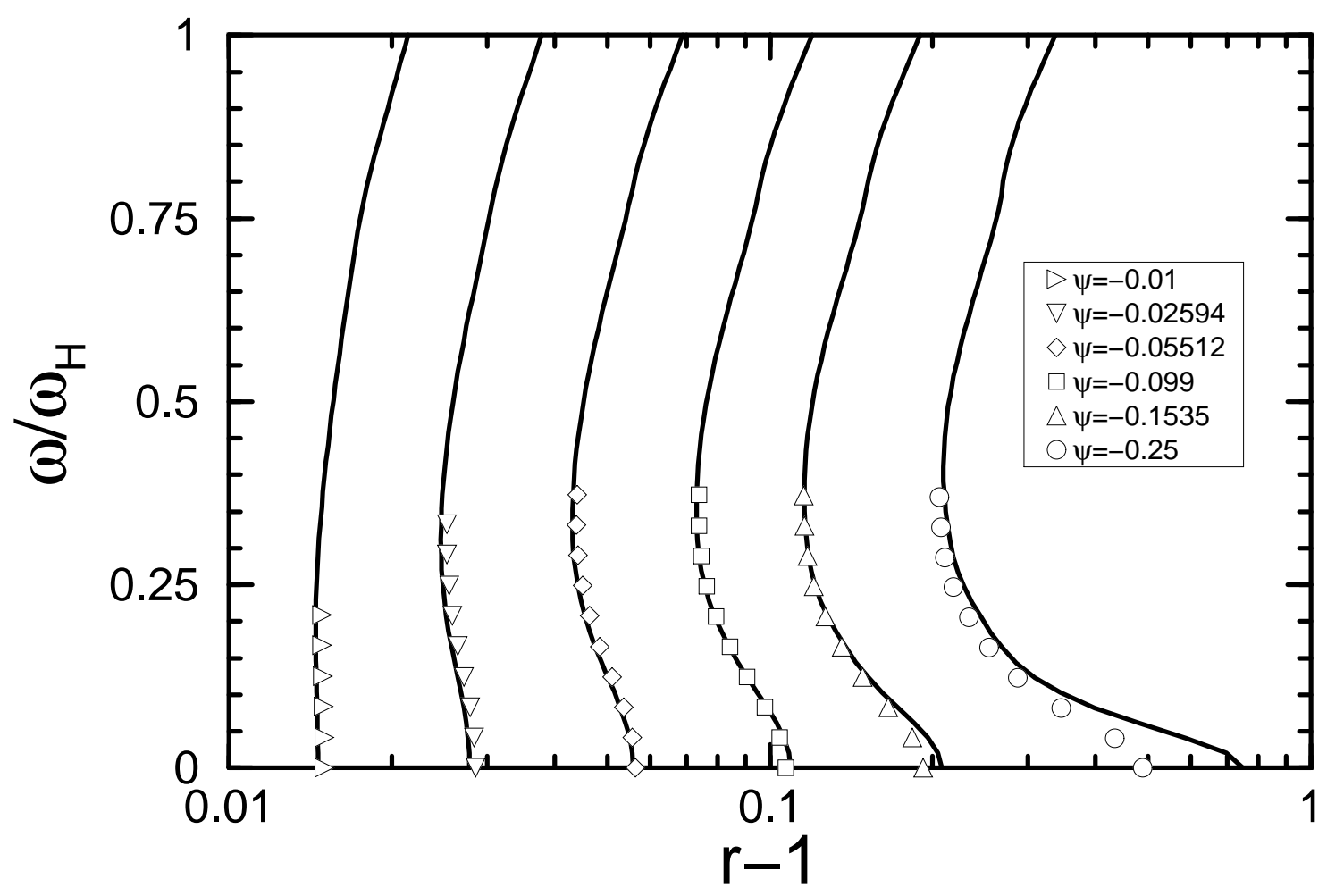

Fig. 12: Hollinger and Lücke, Phys. Rev. E Influence of the Soret effect on convection of binary fluids 